Intensité de l'investissement privé en R\&D dans les pays de l'OCDE : Impact et complémentarité des mesures de soutien financier

Benjamin Montmartin 


\section{GATE Groupe d'Analyse et de Théorie Économique Lyon-St Étienne}

93, chemin des Mouilles 69130 Ecully - France

Tel. +33(0)4 72866060

Fax $+33(0) 472866090$

6, rue Basse des Rives 42023 Saint-Etienne cedex 02 - France

Tel. +33 (0)4 77421960

Fax. +33 (0)4 77421950

Messagerie électronique / Email : gate@gate.cnrs.fr

Téléchargement / Download : http://www.gate.cnrs.fr - Publications / Working Papers 


\title{
Intensité de l'investissement privé en R\&D dans les pays de l'OCDE : Impact et complémentarité des mesures de soutien financier
}

\author{
Benjamin Montmartin*
}

\begin{abstract}
Résumé
Les politiques de soutien financier à la R\&D se sont multipliées depuis les années 80 dans les pays de I'OCDE avec une utilisation croissante des mesures fiscales. Cependant, il existe assez peu d'études mesurant l'impact macroéconomique de ces mesures sur l'investissement privé en R\&D. L'objectif de cet article est d'analyser l'impact et la complémentarité des politiques internes de soutien financier à la R\&D mais aussi l'influence des politiques externes sur l'intensité de la R\&D financée par le secteur privé. En utilisant une base de données couvrant 25 pays de l'OCDE sur la période 1990-2007, nos estimations en panel dynamique montrent que seules les politiques internes de soutien financier indirect (incitations fiscales) influencent significativement l'intensité de la R\&D privée. Si les instruments de soutien direct (subventions et prêts) n'ont pas d'impact significatif, il apparaît que leur renforcement nuirait à l'efficacité des mesures indirectes. Alors que la $R \& D$ publique externe semble dynamiser l'investissement privé en $R \& D$, les politiques extérieures de soutien financier (direct et indirect) ne montrent pas d'influence significative.
\end{abstract}

\begin{abstract}
R\&D financial support policies have proliferated since the 80's in the OECD countries with a growing use of tax incentives. Nevertheless, there are relatively few studies that measure the macroeconomic impact of these policies on private investment in R\&D. The objective of this paper is to analyze the impact and complementarity of national R\&D financial support policies but also to study the influence of foreign policies on Business-funded R\&D intensity. Using a database covering 25 countries of OECD over the period 1990-2007, our dynamic panel data results show that only indirect support policies (tax incentives) affect significantly the Business-funded R\&D intensity. Even though direct financial support policies (subsidies, loans) do not have any significant effect, their strengthening would be detrimental to the effectiveness of indirect support policies. Whereas foreign public R\&D seems to boost private investment in R\&D, foreign financial instruments do not have any significant influence.
\end{abstract}

JEL classification : $\mathrm{H} 25,031,038$

Keywords : R\&D financial support policies, Business-funded R\&D intensity, complementarity, panel data

*Université de Lyon, Lyon, F-69007, France; CNRS, GATE Lyon Saint-Etienne, Ecully, F-69130, France ; Université Jean Monnet, Saint-Etienne, F-42000, France, e-mail : benjamin.montmartin@univ-st-etienne.fr 


\section{Introduction}

La Commission Européenne, dans le cadre de la "Stratégie Europe 2020"1, a fixé un objectif d'investissement en R\&D équivalent à $3 \%$ du PIB dont les $2 / 3$ doivent être financés par le secteur privé. Cet objectif semble toutefois ambitieux au regard de la réalité puisque l'investissement global en R\&D de l'UE a représenté $2 \%$ du PIB en 2009 et l'investissement financé par les entreprises s'est élevé à 1,09\% du PIB (source : Eurostat). Ainsi, si l'écart avec l'objectif d'investissement financé par le secteur public apparaît faible $(0,11$ point de pourcentage), celui avec l'objectif d'investissement financé par le secteur privé apparaît important (0,91 point de pourcentage). En termes de répartition du financement de la $R \& D$, cela implique que la part du financement public (privé) de la R\&D de l'Union Européenne est bien supérieur (inférieur) à son objectif ( $46 \%$ de la R\&D de l'UE est financée par le secteur public pour un objectif de 33\%).

La fixation d'objectifs concernant l'intensité des dépenses de R\&D n'est pas une spécificité européenne puisque l'ensemble des pays de l'OCDE ont fixé de tels objectifs comme le montre le tableau de l'annexe 1. Cela montre que les pouvoirs publics partagent la vision de la littérature économique, à savoir que, du fait de l'existence de nombreuses défaillances de marché qui créent un gap entre rendement privé et rendement social, les firmes sousinvestissent en R\&D par rapport à l'optimum. Cela justifie la mise en place de politiques publiques de soutien à la $R \& D$ privée qui vont permettre d'augmenter le rendement privé ou de réduire le coût de la $R \& D$. Dans le cadre de cet article, nous nous concentrerons plus spécifiquement sur les mesures de soutien financier à la R\&D privée (contrats, subventions, prêts, mesures fiscales).

Cette justification théorique suppose que ces politiques produisent pleinement leurs effets incitatifs sur les décisions d'investissements des firmes en R\&D. Or, en observant les données d'investissements privés en $R \& D$, on peut mettre en doute la capacité de ces politiques à produire des effets incitatifs suffisants. Par exemple, l'intensité de la R\&D financée par le secteur privé de l'UE est passé de 1,03\% du PIB en 1999 à 1,09\% du PIB en 2009 alors que dans de nombreux pays le coût des mesures de soutien financier à la $R \& D$ a nettement augmenté et que les politiques communautaires pour la R\&D ont vu leur dotations fortement augmenter (Programme Cadre de Recherche et Développement et Politique Régionale). De même, on constate que les pays de l'UE dont l'investissement privé en $R \& D$ est au maximum inférieur de 0.2 point de pourcentage au seuil de $2 \%$ (Danemark, Allemagne, Finlande, Suède) sont des pays dont la générosité du système fiscal à la $R \& D$ et les financements directs (contrats, subventions, prêts) sont moins importants que dans la moyenne de I'UE (et de l'OCDE, source: OCDE, calculs de l'auteur).

\footnotetext{
${ }^{1}$ Les objectifs de la "Stratégie Europe 2020" reprennent les objectifs de l'agenda de Lisbonne (2000) concernant la R\&D. Pour toute information, voir : http ://ec.europa.eu/europe2020/index_en.htm
} 
Au-delà de ces constats statistiques, la littérature met également en avant de nombreux éléments qui peuvent limiter l'effet incitatif des politiques de soutien financier à la R\&D privée et/ou générer des effets externes négatifs au niveau macroéconomique. Au niveau microéconomique par exemple, les entreprises peuvent être incitées à substituer (partiellement) les aides publiques ou les gains fiscaux obtenus à leur propre effort de R\&D. Au niveau sectoriel, il est possible que ces politiques génèrent des distortions importantes sur l'allocation du capital conduisant à des effets de substitution entre firmes et secteurs. II se peut également que les stimuli publics génèrent des hausses de coûts des inputs de la $R \& D$ du fait des tensions sur l'offre d'inputs qui seront négatives pour l'investissement privé en R\&D.

Si la littérature empirique cherchant à évaluer l'impact des instruments de soutien financier à la $R \& D$ sur l'investissement privé en $R \& D$ est riche (cf. les revues de David et al. (2000), Hall et Van Reenen (2000), Mairesse et Lentille (2009)), elle a deux principales spécificités comme le soulignent Guellec et al. (2003). D'une part, la grande majorité d'entre elles évaluent la capacité d'un instrument spécifique à exercer un effet d'additionalité sur l'investissement privé en $R \& D$. D'autre part, ces études sont essentiellement menées au niveau microéconomique. Au final, à notre connaissance, il n'existe aujourd'hui que trois études analysant l'impact des mesures de soutien financier direct et indirect au niveau macroéconomique (Guellec et al. (2003), Shin (2006), Falk (2006)) et seule une utilise comme variable dépendante l'intensité de l'investissement privé en $R \& D$.

S'il convient de rappeler que l'impact des politiques de soutien financier sur l'intensité de la R\&D financée par le secteur privé n'est pas le seul objectif de ces mesures, il nous paraît important d'investir cette question trop peu traîtée au niveau macroéconomique. Dans ce cadre, l'objectif de cet article est triple. Dans un premier temps, nous testons la capacité des politiques de soutien direct et indirect à la $R \& D$ privée à influencer positivement l'intensité des dépenses privées de R\&D. Dans un second temps, nous investissons la question de la complémentarité entre instruments de soutien direct et indirect car ces instruments qui reposent sur des mécanismes d'incitations différents pourraient entrer en conflit. Finalement, nous investissons une question peu traitée par la littérature empirique, à savoir, l'existence d'intéractions entre politiques nationales de soutien à la $R \& D$ privée. Cette question est importante car l'internationalisation croissante des activités de R\&D (Hall, 2011) portée notamment par les entreprises multinationales ${ }^{2}$ sous-tend des stratégies d'investissement de plus en plus globales qui vont pouvoir tenir compte des écarts de générosité des systèmes fiscaux et d'aides publiques à la R\&D.

${ }^{2}$ L'OCDE (2011) montre que les multinationales représentent une part substantielle des dépenses de R\&D. Cette part et l'importance de l'internationalisation de la R\&D est d'autant plus forte pour les petits pays exportateurs (Irlande, Belgique, Autriche, République Tchèque) où les filliales de multinationales étrangères représentent plus de la moitié de l'investissement total des entreprises sur le territoire. 
Les estimations réalisées sur 25 pays de l'OCDE durant la période 1990-2007 font apparaître une différence nette entre la capacité des instruments de soutien financier direct et celle des instruments indirects à augmenter l'intensité des dépenses de R\&D financée par le secteur privé. En effet, alors que les mesures de soutien indirect augmentent significativement l'investissement des firmes en $R \& D$, nos résultats montrent une relative neutralité des mesures de soutien direct. Cette différence d'impact apparaît d'autant plus importante que ces deux types d'instruments semblent générer des effets de substitution l'un sur l'autre suggérant une difficulté supplémentaire à prendre en compte dans la définition d'un mix de mesures directes et indirectes.

Par ailleurs, nos résultats ne font pas ressortir d'influence des politiques externes de soutien à la $R \& D$ privée (direct et indirect) sur le niveau d'investissement des firmes dans un pays. Ce résultat combiné avec les précédents semble appuyer les études empiriques rendant compte d'un impact très marginal des aides publiques sur la localisation des activités de $R \& D$ d'une part et d'un impact très faible des mesures fiscales sur l'investissement en R\&D des grandes entreprises qui sont les plus à même de bénéficier des différences de fiscalité. Nos résultats montrent également l'influence marquée des conditions de financement interne et dans une moindre mesure externes sur le niveau d'investissement en R\&D des firmes ce qui laissent penser que le mix de mesures directes pourrait être davantage orienté vers des mesures de financements préférentiels par exemple.

La suite de cet article est organisé de la façon suivante. La section 2 présente les principaux résultats de la littérature (notamment macroéconomique) sur les instruments de soutien financier direct et indirect à la $R \& D$ privée. La section 3 présente les modèles empiriques et les données utilisées. La section 4 propose une analyse statistique et graphique des données. La section 5 expose la méthodologie d'estimation retenue et présente nos résultats. Finalement la section 6 fournit les conclusions de cet article. 


\section{Une vue d'ensemble de l'effet des instruments de soutien financier sur l'investissement privé en $R \& D$}

\subsection{Présentation des instruments de soutien financier à la R\&D privée et des principaux effets limitant leur impact}

Avant de présenter une vue d'ensemble des résultats empiriques fournis par la littérature, nous présentons rapidement les principaux instruments du soutien financier direct et indirect ainsi que les principaux effets pouvant limiter l'impact positif de ces instruments. Classiquement, la littérature décompose les instruments de soutien financier à la $R \& D$ privée en deux catégories:

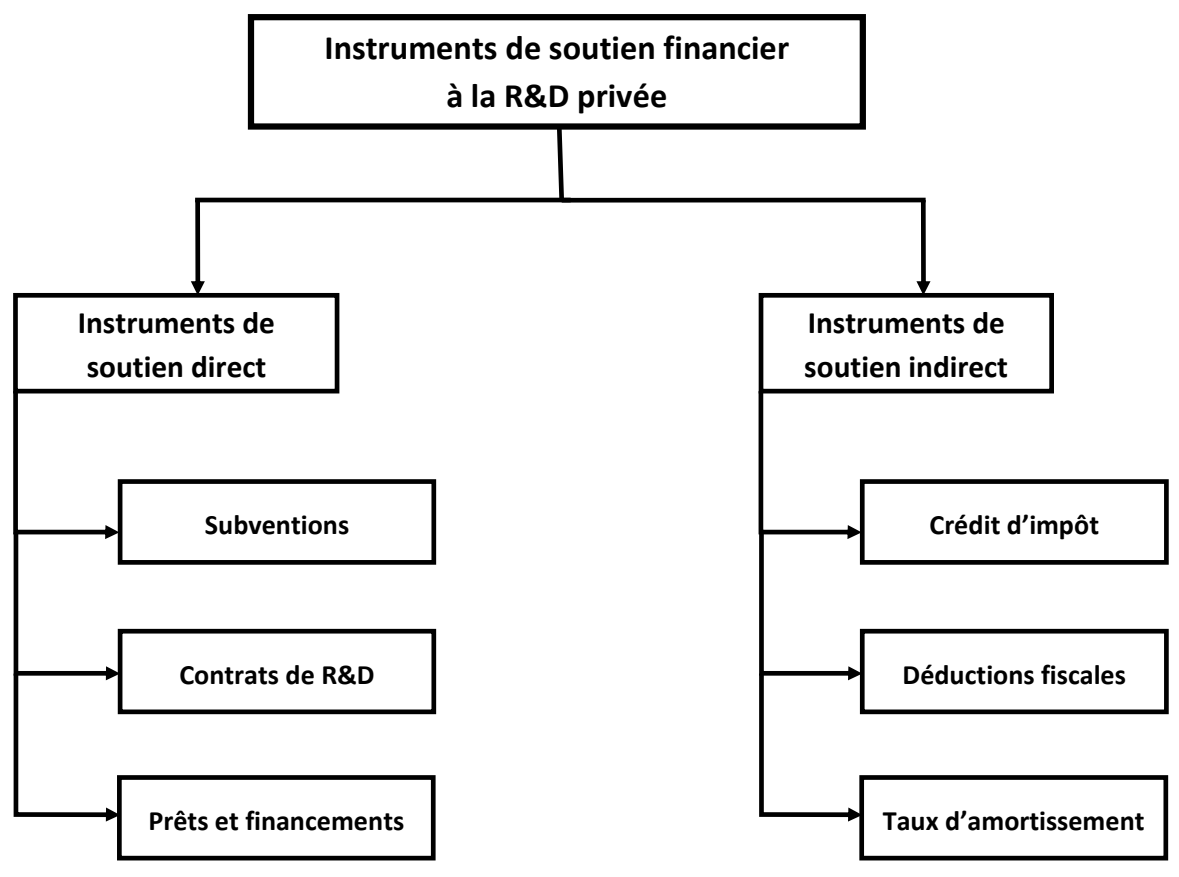

La première catégorie d'instruments dit de soutien direct renvoie à l'ensemble des instruments qui affectent (principalement) le rendement des projets de $R \& D$ et nécessite la mise en place d'une ligne budgétaire dédiée. Ces instruments prennent principalement la forme de contrats de $R \& D^{3}$, de subventions et de prêts. La seconde catégorie d'instruments dit

\footnotetext{
${ }^{3}$ Qui représentent la part la plus importante des mesures de soutien direct selon David et al. (2000) car elle inclus l'ensemble des contrats de R\&D attribués aux entreprises privés du secteur aéronautique et militaire.
} 
de soutien indirect renvoie à l'ensemble des instruments qui affectent (principalement) le coût des projets de $R \& D$ et qui ne nécessitent pas la mise en place d'une ligne budgétaire dédiée. Ces instruments prennent principalement la forme de crédits d'impôt recherche (qui réduisent directement le montant de l'impôt) et de déductions fiscales (qui permettent une réduction du revenu imposable). D'un point de vue général, les mesures concernant les taux d'amortissement constituent un cas spécifique dans le sens où elles ne concernent que les dépenses en capital (équipements et actifs intangibles) car les autres dépenses de R\&D sont amortissables à $100 \%$ dès la première année dans la plupart des pays.

Comme nous l'écrivions en introduction, la justification économique de la mise en place de telles mesures suppose qu'il existe un gap entre investissement courant et optimal mais aussi que ces mesures exercent un effet incitatif suffisant et n'engendrent pas d'effets externes négatifs importants. Si la première condition est généralement admise, les autres le sont plus difficilement. En effet, la littérature (Capron et al. (1997), David et al. (2000), Hall et Van Reenen (2000)) met en avant trois effets principaux pouvant réduire l'effet positif de ces mesures. Au niveau microéconomique, les mesures de soutien direct et indirect peuvent ne servir qu'à financer des projets de $R \& D$ qui auraient été financés sans la mise en place de ces mesures ou ne pas être utilisées pour augmenter les dépenses de R\&D. Dans ce cas, les fonds publics se substituent (partiellement) au financement privé de la $R \& D$. Au niveau sectoriel, les politiques de soutien financier peuvent perturber l'allocation du capital au profit de certaines firmes et/ou secteurs et ainsi avoir un effet global faible voir négatif. En effet, même si les mesures de soutien financier direct et/ou indirect incitent les firmes qui en bénéficient à augmenter leur investissement en $R \& D$, elles peuvent créer des distortions vis à vis des entreprises et secteurs qui n'en bénéficient pas (ou très peu) et réduirent l'investissement de ces derniers en $R \& D^{4}$. Finalement, au niveau macroéconomique, les mesures de soutien financier peuvent créer des tensions sur le prix des inputs de la $R \& D$ et notamment sur le principal, à savoir, le travail. En effet, la mise en place d'un programme important d'aide à la $R \& D$ privée peut se traduire par une augmentation de la demande d'inputs de la $R \& D$. Comme le stock de personnel qualifié pour les activités de R\&D est fixe au moins à court terme, la hausse de la demande va exercer une pression à la hausse des salaires des chercheurs. Cela aura pour conséquence d'augmenter le coût marginal de la $R \& D$ et se traduira, toute chose égale par ailleurs, par une réduction de l'investissement privé en $R \& D$.

\section{Est-ce que ces effets sont aussi importants pour les mesures de soutien direct qu'indirect?}

Selon David et al. (2000, p.502), "Because a tax credit directly reduces the marginal cost of R\&D, one would not expect to see "crowding out" effects on industrial $R \& D$

\footnotetext{
${ }^{4}$ Par exemple, les firmes subventionnées peuvent réussir avec une probabilité plus grande et dans un délai plus court la commercialisation d'innovations ce qui peut réduire le rendement espéré des projets de R\&D des firmes non aidées.
} 
spending except via the channel of an increase in the real cost of $R \& D$ (if the inputs are inelastic supply)". Cette affirmation mériterait cependant d'être acompagnée de précisions concernant les conditions qu'elle suppose. En effet, si un Etat met en place un crédit impôt recherche en volume, celui-ci va bénéficier à l'ensemble des firmes réalisant des activités de $R \& D$ alors que certaines n'augmenteront pas leur investissement en $R \& D$ suite à sa mise en place. En revanche, si l'Etat met en place un crédit d'impôt incrémental, alors les firmes devront augmenter leur effort d'investissement pour en bénéficier. De même, pour que la mise en place de mesures fiscales ne créent pas de distortion entre firmes et secteurs, il faut qu'elles s'appliquent de manière uniforme à l'ensemble des secteurs, qu'elles concernent l'ensemble des dépenses de R\&D et qu'il n'existe pas de seuils. Ainsi, la réponse de David et al. (2000) est valable sous des conditions très restrictives et peu réalistes. De ce fait, il apparaît difficile de savoir si les effets limitant l'efficacité des mesures de soutien indirect sont plus (moins) fortes que ceux limitant l'efficacité des mesures de soutien direct.

La suite de cette section propose une brève présentation des résultats (notamment macroéconomique) de la littérature empirique concernant l'impact des mesures de soutien direct et indirect sur l'investissement des firmes en R\&D. La profonde distinction entre instruments de soutien direct d'une part et indirect d'autre part dans la littérature empirique nous conduit à décomposer cette première section en trois parties. La première présente les résultats des études empiriques portant uniquement sur les instruments de soutien direct. La seconde présente les résultats des études empiriques portant uniquement sur les instruments de soutien indirect. Finalement, la dernière présente les résulats des études macroéconomiques analysant simultanément l'influence des deux types d'instruments.

\subsection{Impact des mesures de soutien financier direct sur la R\&D privée : des résultats contrastés}

La littérature étant riche sur le sujet, il existe plusieurs articles synthétisant les résultats empiriques obtenus (Capron et al. (1997) et David et al. (2000)). Celui de Capron et al. (1997) qui analyse les résultats de 19 études aboutit à la conclusion que, d'un point de vue global, les mesures de soutien public direct génèrent un effet de levier sur la $R \& D$ privée. Cela signifie que l'ensemble des fonds publics reçus sont utilisés pour la $R \& D$ mais aussi que ces fonds ont permis d'augmenter le rendement ou la productivité de la R\&D et ainsi inciter les entreprises à davantage investir. Cependant ce constat très positif doit être relativisé. En effet, comme le souligne Capron et al. (1997), si l'on ne considère que les études microéconomiques alors les résultats sont beaucoup plus contrastés. Par ailleurs, si les études macroéconomiques de Levy et Terleckyj (1983) et de Lichtenberg $(1987)^{5}$

\footnotetext{
${ }^{5}$ Ces deux études sont réalisées sur données américaines.
} 
montrent un effet de levier significatif du soutien public direct, l'étude de Levy (1990) sur 9 pays de l'OCDE montre qu'il existe une forte hétérogénéité entre pays. En effet, ses résultats montrent que si les mesures de soutien direct ont un effet de levier aux Etats-Unis, au Japon, en France, en Allemagne et en Suède, elles semblent avoir un effet de substitution au Pays-Bas et au Royaume-Uni ${ }^{6}$.

Par ailleurs, Capron et al. (1997) soulignent le fait que la majorité de ces études ne prennent pas en compte certaines caractéristiques déterminantes de l'investissement en $R \& D$ et de l'allocation des aides publiques directes à la $R \& D$. La plus importante d'entre elles (au niveau macroéconomique) est l'absence d'une spécification intégrant un processus d'ajustement de l'investissement des firmes en R\&D. Or, les dépenses de R\&D sont essentiellement constituées d'investissements de long terme ce qui fait que le niveau d'investissement antérieur en $R \& D$ est un déterminant premier de l'investissement courant en R\&D. D'ailleurs, Capron et al. (1997) notent que les deux études ${ }^{7}$ introduisant un processus dynamique rendent compte d'un effet de substitution des mesures de soutien public direct. La seconde caractéristique importante non prise en compte concerne l'hypothèse d'exogénéité des mesures de soutien direct. En effet, il est clairement admis que les subventions, contrats et prêts ne sont pas alloués de manière aléatoire par les pouvoirs publics. La non prise en compte de ce biais de sélection peut expliquer la présence d'une relation positive entre $R \& D$ privée et mesures de soutien direct.

Afin de proposer une estimation macroéconomique plus satisfaisante de l'effet des mesures de soutien direct sur l'investissement privé en R\&D, Capron et al. (1997) testent sur 7 pays de l'OCDE une spécification intégrant un processus d'ajustement dynamique ${ }^{8}$. Leurs résultats sont assez différents de ceux de Levy (1990) puisque les aides publiques directes ne semblent générer un effet de levier sur l'investissement privé en R\&D qu'au Royaume-Uni. A l'inverse, un effet de substitution est visible au Canada, en France et en Italie ${ }^{9}$.

Puisque les données utilisées concernant les dépenses de R\&D sont obtenues par aggrégation de données sectorielles, les auteurs testent également l'importance et le signe de l'effet de distortion engendré par ces politiques en estimant l'effet marginal pondéré du soutien public direct ${ }^{10}$. Les résultats obtenus sont nettement différents des précédents ce

\footnotetext{
${ }^{6}$ Par ailleurs, aucun effet marginal significatif n'est présent en Suisse et en Italie.

${ }^{7}$ Nadiri (1980) utilise des données sur les industries américaines produisant des biens durables et Fölster et Trofimov (1996) utilisent des données individuelles sur des entreprises suédoises.

${ }^{8}$ Les dépenses nationales de R\&D sont obtenues en agrégeant les données de l'ensemble des secteurs industriels de chaque pays.

${ }^{9}$ Les mesures de soutien direct ne semblent pas avoir un effet significatif dans les autres pays (Etats-Unis, Allemagne, Japon)

${ }^{10}$ Cela signifie qu'après avoir estimé l'effet marginal pour chaque industrie, ils pondérent ces effets en utilisant la part du soutien direct national allouée à chaque industrie. On obtient donc, pour chaque pays, la moyenne pondérée des effets marginaux de chaque industrie.
} 
qui démontre que les mesures de soutien direct génèrent d'importants effets externes entre industries ${ }^{11}$. Puisque l'effet marginal pondéré des aides publiques directes est supérieur à l'effet obtenu sans pondération, les auteurs concluent que les effets inter-industries sont plutôt négatifs, i.e, les distortions générées par le soutien public entraînent des externalités négatives.

Quelques années plus tard, David et Hall (2000) propose une revue de littérature plus complète tenant compte de 30 études économétriques réalisées entre 1966 et 1999 . Globalement, les conclusions sont similaires à celles de Capron et al. (1997), c'est-à-dire que la majorité des études supportent l'idée d'un effet de levier des instruments de soutien direct. En revanche, si l'on s'intéresse aux seules études microéconomiques, les résultats sont très contrastés puisque la moitié des études montre un effet de levier alors que l'autre moitié montre un effet de substitution. Il est intéressant de noter qu'à ce niveau d'analyse, les méthodes économétriques permettant de tenir compte de l'endogénéité des mesures de soutien direct arrivent toutes à la conclusion d'un effet de substitution (Lichtenberg (1988), Wallsten (1999) et Toivanen et Niininen (1998)). Les résultats des études macroéconomiques convergent quant à elles quasi-unanimement vers l'idée d'un effet de levier. Cependant, David et Hall (2000) soulignent également que les problèmes de spécification et d'estimation de ces études ${ }^{12}$ laissent penser qu'elles sur-estiment l'effet positif des aides publiques directes à la R\&D.

Ainsi, les revues réalisées par Capron et al. (1997) et David et Hall (2000) montrent un certain contraste entre les résultats des études menées au niveau microéconomique et celles menées au niveau macroéconomique. David et Hall (2000) soutiennent l'idée que cette différence pourrait être liée en partie à la non prise en compte (au niveau macroéconomique) de l'impact des politiques de soutien direct sur le coût des inputs de la R\&D. Goolsbee (1998) montre à partir de données américaines qu'une augmentation du soutien direct à la R\&D privée a un effet significatif sur la hausse des salaires des ingénieurs et chercheurs. Selon l'auteur, la non prise en compte de cet effet-prix implique que les estimations macroéconomiques proposées par la littérature sur-estiment de 30 à $50 \%$ l'effet du soutien public direct. L'étude de Wolff et Reinthaler (2008) menée sur un panel de 15 pays de l'OCDE entre 1981 et 2002 semble accréditer cette thèse. En effet, ils montrent que si les instruments de soutien direct ont un effet positif sur les dépenses de R\&D des firmes, ils n'ont pas d'influence significative sur le nombre de chercheurs employés par ces entreprises. Puisque le principal input de la R\&D est le travail, ce résultat suggère que l'effet positif du soutien public direct observé au niveau macroéconomique reflète essentiellement une hausse du salaire des chercheurs.

\footnotetext{
${ }^{11}$ Ces effets externes inter-industries renvoient au fait qu'une subvention accordée à une certaine industrie peut avoir un effet de stimulation ou d'inhibition sur l'investissement en R\&D des autres industries, notamment celles qui sont proches technologiquement ou économiquement

${ }^{12}$ Non prise en compte du problème d'endogénéité des mesures de soutien direct et absence de processus d'ajustement.
} 
Les résultats fournis par la littérature concernant l'impact des mesures de soutien direct sur la R\&D privée sont donc assez ambigus. En effet, au niveau microéconomique, la présence d'un effet de levier ne semble pas être l'hypothèse la plus probable alors que si cette hypothèse semble validée au niveau macroéconomique, la non prise en compte des effets inter-industrie et de l'effet-prix sur les inputs laisse à penser que ces études sur-estiment assez nettement l'effet des politiques de soutien financier direct sur l'investissement des firmes en R\&D. Cette absence de conclusion claire concernant des politiques de soutien direct semble renforcée par la méta-analyse réalisée par Garcia-Quevedo (2004) à partir de 39 études économétriques estimant l'effet du soutien public direct sur l'investissement en $R \& D$ du secteur privé. En effet, en retenant de nombreux éléments ${ }^{13}$ pouvant expliquer les différences de résultats entre ces études économétriques, l'auteur ne parvient pas à les expliquer c'est-à-dire qu'aucune des variables retenues n'est significative.

\subsection{Impact des mesures de soutien financier indirect sur la R\&D privée : un impact significatif}

La revue de littérature réalisée par Hall et Van Reenen (2000) souligne la prépondérance des travaux réalisés au niveau microéconomique à l'image des études portant sur les instruments de soutien direct. Concernant les études sur données américaines où un crédit d'impôt recherche a été mis en place en 1981, il apparaît clairement une rupture entre les résultats obtenus sur des données avant 1983 et ceux obtenus sur des données allant plus loin dans le temps. En effet, les résultats des premières études montrent que l'élasticité-prix des dépenses de $R \& D$ privées est très faible voir non significative. Les analyses coût-bénéfice résultantes concluent donc à une incapacité du crédit d'impôt recherche à exercer un effet de levier sur l'investissement des firmes en R\&D. A l'inverse, les travaux utilisant des données plus récentes estiment que l'élasticité-prix des dépenses de $R \& D$ est de l'ordre de l'unité voir supérieure. Les analyses coût-bénéfice résultant de ces estimations montrent un effet de levier significatif des mesures de soutien indirect puisque pour chaque dollar de recette fiscale perdue, les entreprises ont investi entre 1,3 et $2 \$$ supplémentaires en $R \& D$. Ces études font également apparaître un effet croissant des mesures fiscales au cours du temps qui serait lié au processus d'ajustement partiel des décisions d'investissement. Cela peut expliquer pourquoi les études menées sur les deux premières années de mise en place du crédit d'impôt (1982 et 1983) aux Etats-Unis ne trouvent pas d'effet significatif de la mesure sur les dépenses de R\&D des entreprises. Concernant les études portant sur d'autres pays que les Etats-Unis, Hall et Van Reenen (2000) concluent que dans leur ensemble les résultats obtenus confirment ceux des études américaines, c'est-à-dire que les mesures de

\footnotetext{
${ }^{13}$ Parmis ces élements, l'auteur retient notamment l'année de l'article, l'échelle de l'étude (firme, industrie, pays), l'utilisation d'un processus d'ajustement, la présence d'effets fixes,...
} 
soutien indirect à la R\&D privée exercent plutôt un effet de levier sur l'investissement des firmes en $R \& D$ sur le long terme. Cet effet progressif des mesures fiscales à la R\&D apparaît également dans les résultats obtenus par Lentille et Mairesse (2009). Sur la base de 33 études microéconomiques, les auteurs mettent en avant une corrélation positive entre l'effet estimé du crédit d'impôt recherche par les études à leur période analyse, i.e, plus l'étude est basée sur des données récentes plus l'effet positif des mesures fiscales est important.

L'article de Mohnen et Lokshin (2009) qui propose (entre autre) un résumé des techniques utilisées pour mesurer l'efficacité des mensures fiscales à la $R \& D$ propose également une synthèse des résultats empiriques obtenus après la revue réalisée par Hall et Van Reenen (2000). Cette revue montre que si les études menées au niveau macroéconomique restent minoritaires, elles se développent notamment autour d'approche structurelle permettant de fournir une meilleure appréciation de l'efficacité des politiques en tenant compte de leur effet sur le bien-être. Ces nouvelles études apportent notamment une analyse plus fine de l'effet des mesures fiscales sur l'investissement privé en R\&D. En effet, comme le note Mohnen et Lokshin (2009), les résultats de ces études montrent que si un crédit impôt recherche incrémental génère un effet de levier sur l'investissement privé en $R \& D$ ce n'est pas le cas d'un crédit d'impôt en volume. Ces études plus récentes confirment globalement la capacité des mesures fiscales à générer un effet de levier sur l'investissement privé bien qu'elles soulignent une différence assez nette selon la base d'application de ces mesures.

Parmi les études prises en compte par Mohnen et Lokshin (2009), une seule est réalisée sur un panel de pays de l'OCDE, à savoir, celle de Bloom et al. (2002). L'objectif de cette dernière est d'estimer l'effet des incitations fiscales à la $R \& D$ sur les dépenses de $R \& D$ privée $^{14}$ de 9 pays de l'OCDE entre 1979 et 1996 . Les auteurs testent différentes spécifications qui intègrent un processus dynamique et des effets fixes temporels et individuels. Leurs estimations évaluent l'élasticité-prix des dépenses de R\&D entre -0.124 et -0.144 à court terme et supérieur à -1 sur le long terme (car le processus d'ajustement de l'investissement privé en $R \& D$ est relativement long). Ces résultats semblent robustes puisqu'en réalisant des estimations individuelles sur chaque pays, les auteurs mettent en évidence un impact positif des mesures fiscales pour sept des neuf pays inclus dans l'étude.

Ainsi, les études empiriques analysant l'effet des mesures fiscales à la R\&D sur l'investissement privé en $R \& D$ arrivent globalement à la conclusion d'une relative efficacité. Cependant cette conclusion doit être prise avec précaution puisque, comme le suggèrent Mohnen et Lokshin (2009), la capacité des mesures de crédit d'impôt à exercer un effet de levier sur l'investissement privé dépend fortement de leur base d'application. Par ailleurs, les études empiriques utilisant une spécification dynamique montrent que les incitations fiscales exercent un effet croissant dans le temps sur la $R \& D$ privée car le processus d'ajustement de l'investissement privé est relativement lent.

\footnotetext{
${ }^{14}$ Les auteurs n'utilisent que les dépenses de R\&D des secteurs industriels de chaque pays
} 


\subsection{Les études macroéconomiques mesurant simultanément les effets des instruments de soutien financier direct et indirect}

Très peu d'études analysent simultanément les effets des politiques de soutien direct et indirect à la $R \& D$ privée au niveau macroéconomique. Pourtant ces études présentent un grand intérêt car elles vont permettre, entre autre, d'analyser la complémentarité des instruments pour augmenter l'investissement privé en R\&D. Cependant, seule l'étude Guellec et al.(2003) analyse explicitement cette complémentarité en introduisant une variable croisée des politiques de soutien financier.

A notre connaissance, l'étude de Guellec et al. (2003) est la première à analyser simultanément l'effet des mesures de soutien direct et indirect à la R\&D privée. L'étude menée sur un panel de 17 pays de l'OCDE sur la période 1981-1996 montre que les mesures de soutien direct et indirect à la R\&D privée ont un effet significativement positif sur l'investissement des entreprises en R\&D. L'impact marginal d'un dollar de soutien direct est ainsi estimé à $0,7 \$$ alors que l'élasticité-prix de long terme des dépenses privées de $R \& D$ est estimé à -0.31 . Par rapport aux résultats de la littérature, l'effet estimé du soutien direct apparaît plus important alors que l'inverse est vrai pour le soutien indirect. Ces résultats sont selon nous fortement liés à la méthode d'estimation utilisée par les auteurs qui mesure l'impact des mesures de soutien financier sur la croissance de l'investissement privé en R\&D et non sur son niveau absolu. Guellec et al. (2003) analysent également la complémentarité des instruments de soutien financier direct et indirect pour dynamiser l'investissement privé en $R \& D$. Leurs résultats montrent que les mesures de soutien direct ont un effet d'éviction important sur les mesures de soutien indirect (et vice-versa), i.e, l'augmentation des mesures de soutien direct va réduire l'effet positif des incitations fiscales sur l'investissement privé en $R \& D$ et vice-versa. Ce résultat montre donc l'importance pour les pouvoirs publics d'avoir une approche globale dans la définition de leur policy-mix de mesures directes et indirectes.

L'étude de Shin (2006) analyse l'influence des mesures de soutien direct et indirect sur les dépenses de R\&D des firmes coréennes entre 1982 et 2002. Les résultats obtenus soutiennent ceux de Guellec et al. (2003) puisque les instruments directs et indirects influencent significativement et positivement l'investissement des firmes en R\&D. Cependant, l'effet estimé des mesures de soutien direct et indirect est plus proche des estimations de la littérature puisque l'effet marginal estimé des aides directes est compris entre 0.1 et 0.15 et que l'élasticité-prix estimé de la R\&D est proche de -1 sur le long terme.

La seule étude macroéconomique utilisant comme variable dépendante l'intensité des dépenses de R\&D privée est celle proposée par Falk (2006). Dans cette étude, l'auteur évalue (entre autre) l'impact des mesures de soutien financier direct et indirect en utilisant 
un panel de 21 pays de l'OCDE entre 1975 et 2002. Les résultats montrent que les mesures de soutien direct $n$ 'influencent pas significativement l'intensité de la $R \& D$ privée alors que les mesures de soutien indirect l'affectent significativement avec une élasticité-prix de la R\&D proche ou supérieure à -1 sur le long terme. A l'image de Wolff et Reinthaler (2008) qui utilisent une mesure relative des aides directes au lieu de leur montant absolu afin de mieux contrôler l'effet-prix sur les inputs de la $R \& D^{15}$, nous pensons que l'absence d'influence des mesures de soutien direct (qui sont exprimées en \% du PIB) dans l'étude de Falk (2006) est liée à une meilleure prise en compte de l'effet-prix sur les inputs de la R\&D par rapport aux précédentes études macroéconomiques.

Ces trois études intégrant à la fois des mesures du soutien financier direct et indirect à la R\&D fournissent finalement des conclusions assez proches des études analysant un instrument en particulier. En effet, ces études font ressortir un effet significativement positif des mesures fiscales sur l'investissement privé en R\&D alors que l'effet des mesures de soutien direct semble moins net bien que seul Falk (2006) ne rend pas compte d'un effet positif. Par ailleurs, l'étude de Guellec et al. (2003) montre l'existence d'un effet d'éviction entre les instruments de soutien direct et indirect.

Cette revue des résultats fournis par la littérature nous a permis de mettre en évidence une différence importante entre les études menées au niveau microéconomique qui sont plus reservées sur l'impact des politiques de soutien à la $R \& D$ privée (instruments directs et indirects) et les études macroéconomiques qui rendent davantage compte d'effets additionnels. Cela nous a également permis de voir que d'un point de vue général, la capacité des mesures de soutien indirect à générer un effet de levier sur l'investissement privé en $R \& D$ semble plus importante que celle des mesures de soutien direct. Finalement, cette revue montre la nécessité d'utiliser un modèle empirique tenant compte de la forte irréversibilité des investissements en $R \& D$ dus à d'importants des coûts d'ajustement.

\subsection{L'évaluation des instruments de soutien financier à la R\&D privée : complexité et objectifs}

Même si dans le cadre de cet article, notre ambition n'est pas de proposer une évaluation des politiques de soutien à la $R \& D$ privée, il nous semble important de présenter très succintement les avantages et inconvénients des instruments de soutien direct et indirect afin de replacer notre propos et nos résultats dans une réalité et un contexte plus large. Une évaluation complète des politiques de soutien à la R\&D privée doit prendre en compte,

\footnotetext{
${ }^{15}$ Et ainsi proposer une estimation plus réaliste de l'effet des mesures de soutien direct.
} 
en plus des effets sur l'investissement en R\&D des firmes, les coûts d'administration induits et surtout leur impact sur le bien-être. Ces considérations plus larges amènent souvent économistes et experts à considérer les instruments de soutien direct et indirect comme des outils complémentaires pour réduire le gap entre investissement effectif et optimal en $R \& D$ car ils reposent sur des mécanismes incitatifs différents.

Les économistes mettent souvent en avant le côté "friendly market" des mesures fiscales qui laissent les décisions d'investissements en $R \& D$ et leur gestion aux entreprises et peuvent bénéficier potentiellement à l'ensemble des firmes réalisant de la $R \& D$ contrairement aux aides directes. Par ailleurs, il est souvent reproché aux aides directes de ne pas être allouées de façon optimale et d'être coûteuses en termes de gestion. Cependant, si les mesures fiscales ont des avantages par rapport aux mesures de soutien direct, de nombreux économistes soulignent leurs limites notamment en termes d'impact potentiel sur le bien-être. En effet, les mesures d'incitation à la $R \& D$ privée doivent promouvoir la réalisation de projets pour lesquels l'écart entre rendement social et rendement privé est le plus important. Or, il est clair que d'un point de vue microéconomique, les firmes vont financer les projets qui ont les rendements privés les plus importants et non les projets où l'écart entre rendement social et privé est le plus significatif. A l'inverse, les aides directes qui sont en partie allouées par une mise en concurrence de projets de R\&D sur des domaines ou sujets prédéfinis par les autorités publiques devraient permettre de mieux cibler les projets ayant un rendement social important.

La littérature économique a mis en évidence de nombreux autres éléments montrant une certaine forme de complémentarité entre mesures de soutien direct et indirect. Cette complémentarité est plus visible lorsque l'on considère d'autres objectifs que l'augmentation des dépenses privées de R\&D et que l'on prend en compte les différences d'incitations entre mesures de soutien direct et indirect. Le tableau ci-dessous qui est inspiré de celui de Carvalho (2011) complète ce début de discussion en proposant un résumé des principaux avantages et inconvénients des instruments de soutien direct et indirect. 


\begin{tabular}{|c|c|c|}
\hline & Avantages & Inconvénients \\
\hline 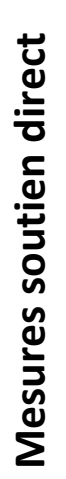 & $\begin{array}{l}\text { - Adaptées pour cibler les activités et projets pour lesquels } \\
\text { l'écart entre rendement privé et social est important } \\
\text { - Théoriquement, la concurrence entre firmes assure que les } \\
\text { fonds publics sont utilisés pour les meilleurs projets de R\&D } \\
\text { - Peut être utilisé pour réduire les effets du cycle économique } \\
\text { sur l'investissement des firmes en R\&D } \\
\text { - Peut encourager la coopération et le transfert de technologies } \\
\text { et ainsi renforcer les externalités de connaissance } \\
\text { - Permet un contrôle du coût des mesures } \\
\text { - Peut constituer un effet de réputation pour les firmes financées } \\
\text { et ainsi réduire leur coût du capital (PME) }\end{array}$ & $\begin{array}{l}\text { - Coûts administratifs élevés pour les firmes et les pouvoirs publics } \\
\text { - Impossible à implémenter pour un nombre très important de } \\
\text { projets } \\
\text { - Cause des distorsions de marchés dans l'allocation des ressources } \\
\text { entre différents domaines de R\&D et entre firmes. } \\
\text { - Sélection des projets a tendance à récompenser les lobbies. La } \\
\text { pression liée aux objectifs de résultats des politiques mises en } \\
\text { place induit un risque de sélectionner des projets ayant de forte } \\
\text { probabilité de réussir, i.e., des projets à fort rendement privé qui } \\
\text { auraient été menés sans aide publique } \\
\text { - Nombreuses sources d'éviction potentielle car les mesures de } \\
\text { soutien direct sont ciblées et affectent le rendement de la R\&D. }\end{array}$ \\
\hline 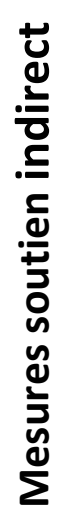 & $\begin{array}{l}\text { - Mesures plus neutres car encourage l'investissement en R\&D } \\
\text { pour l'ensemble des firmes (même s'il peut également être } \\
\text { ciblé sur certains secteurs) et notamment les PME } \\
\text { - Les entreprises décident des projets sur lesquels investir } \\
\text { - Réduit le risque de marchés publics truqués } \\
\text { - Ne nécessite pas de mettre à disposition une ligne de budget } \\
\text { car le coût ne s'exprime qu'en perte de revenu fiscal } \\
\text { - Coûts de mise en place et de gestion relativement faible (bien } \\
\text { que l'OCDE (2001) tempère cela) } \\
\text { - Les mesures fiscales réduisent directement le coût de la R\&D ce } \\
\text { qui réduit théoriquement les sources d'éviction potentielles }\end{array}$ & $\begin{array}{l}\text { - Difficile de contrôler le coût des mesures } \\
\text { - Effets limités pour les firmes ne réalisant pas assez de profit ou } \\
\text { réalisant des investissements trop importants en R\&D (grandes } \\
\text { entreprises) car elles ne bénéficient pas au maximum des mesures } \\
\text { fiscales } \\
\text { - Un risque d'éviction non négligeable car ces mesures peuvent } \\
\text { réduire le coût de projets qui auraient de toute façon été menés } \\
\text { (notamment dans le cas d'un crédit d'impôt en volume) } \\
\text { - Les incitations fiscales vont tendre à favoriser les projets de R\&D } \\
\text { ayant le rendement privé le plus élevé à court terme. Ainsi, les } \\
\text { projets avec un rendement social élevé et long ne seront pas } \\
\text { favorisés par ce type de mesure. } \\
\text { - Puisque les firmes décident des projets et que la coopération est } \\
\text { très rarement une condition d'éligibilité, les externalités de } \\
\text { connaissances générées devraient être faibles. }\end{array}$ \\
\hline
\end{tabular}




\section{Modèles empiriques et présentation des données}

\subsection{Les modèles empiriques}

Pour mesurer l'effet des mesures de soutien direct et indirect à la R\&D sur l'intensité de l'investissement privé en $R \& D$, notre modèle s'inspire des modèles empiriques d'investissement privé en R\&D développé par David et al. (2000), Guellec et al.(2003), Shin (2006) et Falk (2006) :

$$
\begin{aligned}
D I R D F I P I B_{i t}= & f\left(I N T E R E T L T_{i t}, C R E D I T P I B_{i t}, D I R D P U B P I B_{i t},\right. \\
& \left.S U B P I B_{i t}, B I N D E X_{i t-1}\right)
\end{aligned}
$$

où l'indice $i$ renvoie au $i^{e}$ pays et l'indice $t$ renvoie à la $t^{e}$ période. DIRDFIPIB $B_{i t}$ représente les dépenses de $R \& D$ financées par le secteur privé en pourcentage du PIB. $I N T E R E T L T_{i t}$ représente le taux d'intérêt à long terme exprimé en pourcentage.

$C R E D I T P I B_{i t}$ représente le montant de crédits accordé au secteur privé exprimé en pourcentage du PIB. DIRDPUBPIB $B_{i t}$ représente la R\&D exécutée par le secteur de l'Etat (hors enseignement supérieur) en pourcentage du PIB. SUBPIB $B_{i t}$ représente le montant des aides directes perçues par les entreprises exprimées en pourcentage du PIB. Finalement, $B I N D E X_{i t-1}$ représente une mesure de la générosité du système fiscal à la $R \& D$ tenant compte des différents instruments de soutien indirect à la $R \& D$ privée ${ }^{16}$. Cette variable est décalée d'une période pour tenir compte du temps d'adaptation du comportement des firmes à des changements de fiscalité sur la R\&D (Guellec et al. (2003) et Shin (2006)). En utilisant une mesure relative du soutien direct et de l'investissement privé en $R \& D$, nous pensons mieux contrôler l'effet des aides directes sur le prix des inputs et ainsi proposer une mesure plus juste de l'impact macroéconomique de ces instruments.

En suivant Shin (2006) et Falk (2006), nous définissons l'équation de l'intensité optimale (ou cible) de l'investissement privé en R\&D comme :

$$
\begin{aligned}
\ln \operatorname{DIRDFIPIB}_{i t}^{*}= & \alpha^{*}+b_{1}^{*} \ln I N T E R E T L T_{i, t}+b_{2}^{*} \ln C R E D I T P I B_{i, t} \\
& +b_{3}^{*} \ln \ln \operatorname{RPUBPIB} B_{i, t}+b_{4}^{*} \ln S U B P I B_{i, t} \\
& +b_{5}^{*} \ln B I N D E X_{i, t-1}+e_{i, t}
\end{aligned}
$$

L'existence de nombreuses défaillances de marché implique une forte probabilité que l'intensité observée des dépenses privée de $R \& D$ diffère de son niveau cible/optimal. Les activités et investissements en $R \& D$ ont des caractéristiques différentes des autres investissements

\footnotetext{
${ }^{16}$ Cet indicateur a été développé par McFetridge et Warda (1983). Plus sa valeur est faible, plus le système fiscal est favorable. Une description plus complète du B-index, de son calcul et de ces hypothèses est proposée en annexe 2 .
} 
notamment en termes d'irreversabilité et de durée qui génèrent d'importants coûts d'ajustement. Cela implique que les firmes ne vont adapter que partiellement leur comportement d'investissement en $R \& D$ aux changements de politiques publiques et de l'environnement économique. Cela nous conduit à retenir le processus d'ajustement partiel suivant :

$$
\begin{aligned}
& \ln \operatorname{DIRDFIPIB}_{i, t}-\ln \text { DIRDFIPIB } B_{i, t-1}= \\
& \theta\left(\ln D I R D F I P I B_{i, t}^{*}-\ln D I R D F I P I B_{i, t-1}\right)
\end{aligned}
$$

où $0 \leq \theta \leq 1$ représente le coefficient d'ajustement de l'intensité courante des dépenses privées de R\&D par rapport à son niveau optimal/cible. Notons que la forme d'ajustement utilisée signifie que l'évolution de l'intensité de l'investissement privé en $R \& D$ sur une période correspond à une fraction de l'écart entre l'intensité optimale et l'intensité observée à la période précédente. Lorsque $\theta=1$ l'ajustement est total en une période alors que si $\theta=0$, il n'y a pas d'ajustement. En insérant l'équation (2) dans (3), on obtient:

$$
\begin{aligned}
\ln \text { IIRDFIPIB }_{i t}= & \alpha+\rho \ln \text { DIRDFIPIB } B_{i t}+b_{1} \ln I N T E R E T L T_{i, t} \\
& +b_{2} \ln C \operatorname{REDITPI} B_{i, t}+b_{3} \ln \text { DIRDPUBPIB } B_{i, t} \\
& +b_{4} \ln S U B P I B_{i, t}+b_{5} \ln B I N D E X_{i, t-1}+e_{i, t}
\end{aligned}
$$

où $\rho=(1-\theta), b_{i}=\theta b_{i}^{*}$ et $\alpha=\theta \alpha^{*}$. Afin de tenir compte de l'influence de variables omises, des spécificités nationales ainsi que des effets du cycle économique, nous ajoutons à l'équation (4) des effets fixes individuel et temporel :

$$
\begin{aligned}
\ln \operatorname{DIRDFIPIB}_{i, t}= & \beta_{i}+\rho \ln \text { DIRDFIPIB } B_{i, t}+b_{1} \ln \operatorname{INTERETLT}_{i, t} \\
& +b_{2} \ln C \operatorname{REDITPI} B_{i, t}+b_{3} \ln \text { DIRDPUBPIB } B_{i, t} \\
& +b_{4} \ln \ln B P I B_{i, t}+b_{5} \ln B I N D E X_{i, t-1}+\tau_{t}+e_{i, t}
\end{aligned}
$$

Les paramètres $b_{i}$ représentent les élasticités de court terme et $b_{i} /(1-\rho)$ les élasticités de long terme. Par simplification, nous réécrivons le modèle (5) comme:

$$
\ln Y_{i t}=\rho \ln Y_{i, t-1}+\beta^{\prime} \ln X_{i, t}+\alpha_{i}+\tau_{t}+e_{i, t}
$$

L'internationalisation croissante des activités de R\&D (Hall, 2011) montre que la localisation des activités de R\&D est une variable stratégique pour les grandes firmes. Ainsi, les investissements en $R \& D$ dans un pays ne vont pas dépendre uniquement des caractéristiques d'un pays mais également de celles des autres pays. Même si la littérature sur la localisation des investissements en $R \& D$ ne met pas en avant les instruments de soutien à la $R \& D$ privée comme un déterminant important, plusieurs auteurs soulignent leur rôle potentiel (Hines (1993, 1994), Thursby (2006), Hall (2011)). Par ailleurs, comme le souligne Hall (2011), aucune des études sur la localisation des activités de R\&D n'intègre explicitement de données sur la fiscalité de la R\&D. Afin d'estimer l'influence des politiques externes de 
soutien à la $R \& D$ sur l'investissement des firmes en $R \& D$ dans un pays, nous étendons le modèle (6) de la façon suivante :

$$
Y_{i, t}=\rho Y_{i, t-1}+\beta^{\prime} X_{i, t}+\gamma^{\prime} W X_{i, t}+\alpha_{i}+\tau_{t}+e_{i, t}
$$

où $W$ est une matrice de pondération spatiale normalisée qui donne une mesure de l'intensité des relations d'un pays $i$ avec les autres pays $j \neq i$. Le choix de la mesure de l'intensité des relations entre pays est toujours cruciale et délicate. Dans notre cas, il s'agit d'obtenir une mesure qui va permettre de rendre compte de l'importance relative des caractéristiques des pays $j \neq i$ sur les décisions d'investissement en $R \& D$ des firmes localisées dans le pays $i$. Comme il est impossible de mesurer correctement cette importance relative qui dépend de nombreux paramètres, nous utilisons trois mesures différentes de l'intensité des relations entre pays. Cela nous permettra d'une part d'augmenter nos chances d'avoir une mesure pertinente de l'intensité des relations entre les pays et d'autre part de comparer l'influence de la mesure retenue sur les résultats.

La première matrice de pondération spatiale utilise des données du commerce bilatéral et définit l'intensité des relations entre le pays $i$ et $j$ comme :

$$
W_{i j}=\frac{1}{2|T|} \sum_{t \in T}\left(\frac{X_{i j}^{t}}{\sum_{j} X_{i j}^{t}}+\frac{M_{i j}^{t}}{\sum_{j} M_{i j}^{t}}\right)
$$

où $X_{i j}^{t}$ représente le montant total des exportations du pays $i$ vers le pays $j$ à la période $t$ et $M_{i j}^{t}$ représente le montant des importations du pays $i$ en provenance du pays $j$. Ainsi, l'intensité des relations entre deux pays est mesurée par la moyenne de l'importance relative de leur commerce bilatéral sur les périodes $T$. Comme nous ne disposons pas de données complètes pour chaque année de notre période d'étude, la moyenne est calculée à partir de l'intensité des échanges bilatéraux observés en $T=\{1995,2000,2005,2008\}$. Les données du commerce bilatéral (qui concerne l'ensemble des secteurs industriels) proviennent de la base STAN de I'OCDE.

La seconde matrice de pondération spatiale essaie de rendre compte de l'intensité des relations technologiques entre pays en utilisant des données de collaborations dans les demandes de brevets internationaux PCT. L'intensité des collaborations technologiques entre le pays $i$ et $j$ est définie comme:

$$
W_{i j}=\frac{\frac{1}{|T|} \sum_{t \in T} b_{i j}^{t}}{\sum_{j} \frac{1}{|T|} \sum_{t \in T} b_{i j}^{t}}
$$


où $b_{i j}^{t}$ représente le nombre de collaborations dans les demandes de brevets $\mathrm{PCT}$ entre le pays $i$ et $j$ à la période $t$. Ainsi, l'intensité des relations entre deux pays est mesurée par la moyenne de l'importance relative de leur collaboration dans les demandes de brevets internationaux au cours de la période d'étude. Les données utilisées sont extraites de la base REGPAT de l'OCDE et nous disposons des données annuelles pour l'ensemble de notre période, c'est-à-dire que $T=18$.

Finalement, nous utilisons une matrice de poids spatiale basée sur la distance géographique entre pays. En utilisant des données sur la distance à vol d'oiseau entre le centre géographique de chaque pays, nous définissons l'intensité des relations entre le pays $i$ et $j$ comme :

$$
W_{i j}=\frac{\frac{1}{d_{i j}}}{\sum_{j} \frac{1}{d_{i j}}}
$$

où $d_{i j}$ représente la distance à vol d'oiseau entre le centre géographique du pays $i$ et celui du pays $j$. Ainsi, l'intensité des relations entre deux pays est mesurée par l'inverse de la distance relative entre leur centre géographique respectif. Les données de distance ont été obtenues à partir de la base EuroBoundaryMap fournit par Eurogeographics.

\subsection{Présentation des données}

Les données utilisées proviennent essentiellement de l'OCDE et du FMI à l'exception de celles concernant le B-index. Ces dernières ont été collectées à partir de l'article de Thomson (2009) qui propose une valeur du B-index pour 25 pays de l'OCDE sur la période 1980-2006. Nous avons préféré les calculs du B-index proposés par Thomson (2009) à ceux proposés par l'OCDE car les calculs du B-index effectués par Thomson (2009) mesure davantage la subvention fiscale effective moyenne par dollar investi en $R \& D$ que la subvention maximale comme le propose l'OCDE. Nous renvoyons les lecteurs à l'article de Thomson (2009) pour connaître l'ensemble des détails concernant ses calculs du B-index. Un bon exemple des différences de mesures induite par l'approche de l'OCDE et celle de Thomson (2009) concerne notamment les mesures fiscales soumises à des conditions d'éligibilité très spécifiques. Par exemple, le Danemark a mis en place en 2002 une déduction de 150\% des dépenses de recherche fondamentale réalisées en collaboration avec les établissements d'enseignement supérieur. Selon le calcul du B-index fourni par l'OCDE, cette mesure est censée représenter une subvention fiscale par $\$$ investi en $R \& D$ de $0,16 \$$ en 2006 . En revanche, selon Thomson (2009), cette mesure est trop spécifique pour être intégrée dans le calcul du B-index. 
La base de données utilisée couvre 25 pays de l'OCDE sur 18 années (1990-2007). Cependant, pour l'ensemble des variables à l'exception du B-index, des données étaient manquantes. Ainsi, le pourcentage de données manquantes de la variable la moins bien renseignée ${ }^{17}$ était de $18 \%$ alors que celui des mieux renseignées (hors B-index) ${ }^{18}$ était inférieur à $2 \%$ avec une moyenne située à $10 \%$. Ces données manquantes sont principalement liées aux particularités des systèmes nationaux de recueil d'informations sur la recherche et développement. En effet, l'Australie, la Grèce (jusqu'en 2003), la Nouvelle-Zélande, la Norvège (jusqu'en 2001) et la Suède (jusqu'en 2003) réalisent (ou réalisaient) des enquètes et statistiques concernant la R\&D tous les deux ans. D'autres pays comme l'Autriche (1993), la Corée (1995), le Mexique (1993) ou la République Tchèque (1995) n'ont mis en place un recueil de statistiques sur la R\&D qu'après 1990.

Pour réduire le nombre de données manquantes, nous avons estimé les données manquantes des pays ayant un système de recueil de données bisannuel en utilisant la moyenne de l'information à $t-1$ et $t+1$ pour déterminer la valeur de la période $t$. En revanche, les informations manquantes des pays qui ont mis en place tardivement des systèmes de recueil d'information sur la R\&D n'ont pas été estimées. Ainsi, notre base de données est un panel non cylindré où $\bar{T}=17.1$, c'est-à-dire qu'en moyenne, nous avons les données pays sur 17,1 années sur une période totale de 18 années ce qui signifie que le non cylindrage est faible. Le pays pour lequel nous avons le moins d'informations est la Corée avec des données disponibles pour 13 années seulement (1995-2007). Par ailleurs, le tableau 1 de l'annexe 3 présente la distribution du nombre de pays présents par année.

\section{Statistiques descriptives et analyse graphique}

\subsection{Description statistique des données}

Les tableaux 2 et 3 de l'annexe 3 fournissent les statistiques descriptives de nos séries ainsi que le tableau des corrélations entre variables. Les statistiques descriptives rendent compte d'une forte hétérogénité entre pays. En effet, l'écart-type de chaque variable (à l'exception du B-index) représente plus de $50 \%$ de leur valeur moyenne. Plus concrètement, cela se traduit par un grand écart entre les valeurs extrêmes. Ainsi, la moyenne de l'intensité des dépenses de R\&D financées par le secteur privé est très proche de $1 \%$ du PIB avec un minimum de $0,03 \%$ et un maximum avoisinant $3 \%$. La moyenne des dépenses de R\&D exécutées par le secteur de l'Etat est proche de $0,25 \%$ du PIB avec un minimum de 0,07\% et un maximum de $0,56 \%$. Les variables financières utilisées montrent également de très

\footnotetext{
${ }^{17} \mathrm{~A}$ savoir le pourcentage de la R\&D financée par le secteur des entreprises.

${ }^{18} \mathrm{~A}$ savoir les données concernant les crédits accordés au secteur privé.
} 
fortes disparités. En effet, si les crédits accordés au secteur privé s'élève en moyenne à $91 \%$ du PIB, ils atteignent un maximum de $231 \%$ et un minimum de $15 \%$. Concernant le taux d'intérêt à long terme, sa moyenne se situe à $7,8 \%$ avec des valeurs extrèmes de $1 \%$ et $46 \%$. Les variables de politiques publiques de soutien à la $R \& D$ sont aussi marquées par d'importants écarts. Si, en moyenne, le soutien financier direct avoisine $0.08 \%$ du PIB, son maximum atteint $0.45 \%$ et son minimum est proche de $0 \%$. La valeur du B-index est quant à elle comprise entre 0.57 et 1.08 avec une moyenne située à 0.95 .

Le tableau 3 qui présente les corrélations entre les variables montre que nous ne devrions pas avoir de problème de multicolinéarité. En effet, la corrélation partielle la plus élevée concerne la relation entre les deux variables financières (crédit et taux d'intérêt) et s'élève seulement à -0.54 . Il est intéressant de noter que la corrélation entre les variables de soutien direct et indirect est très faible $(0.17)$ ce qui exclut a priori une relation positive et forte entre ces deux variables.

\subsection{L'analyse graphique des données}

Afin d'avoir une connaissance générale de l'évolution de nos variables sur la période d'étude, nous analysons graphiquement leur tendance. Les graphiques d'évolutions de chacune des variables du modèle sont disponibles en annexe 4.

\section{Dynamique de la variable dépendante au sein de l'OCDE}

Concernant la variable dépendante, le graphique 1 montre une tendance à l'augmentation de l'intensité des dépenses de R\&D financées par les entreprises sur la période à l'exception de certains pays européens comme le Royaume-Uni, I'Italie, la Pologne, les Pays-Bas, la Hongrie, la Norvège et la Belgique. Certains pays ont connu une très forte croissance de l'investissement privé en R\&D et notamment la Corée, le Danemark, la Finlande, l'Australie ou encore l'Autriche. On remarque également le cas atypique de la Suède qui a connu une très forte progression de l'investissement en R\&D financé par le secteur privé jusqu'à la crise internet de 2001 qui marque un point d'inflexion dans cette dynamique. Ainsi, la R\&D financée par le secteur privé en Suède est passé de $1.66 \%$ du PIB en 1991 à 2,96\% en 2001 puis a reculé pour s'établir à 2,11\% en 2007.

Le graphique 2 qui représente la part de la $R \& D$ totale financée par le secteur privé montre une tendance moins nette que celle observée précédemment bien que d'une manière générale, les pays dont l'intensité de la $R \& D$ privée a augmenté (diminué) ont connu une augmentation (diminution) de la part de la $R \& D$ financée par le secteur privé. Certains pays comme la Corée, l'Autriche, la République Tchèque et l'Espagne dont l'intensité de la R\&D financée par le secteur privé a augmenté ont pourtant vu la part de la R\&D financée par le 
secteur privé légèrement reculée. Notons qu'il existe des cas très spécifiques où l'évolution du financement privé de la $R \& D$ a été très marquée. Ainsi la Pologne, la République Tchèque et dans une moindre mesure l'Irlande qui ont vu la contribution du secteur privé à l'effort de $R \& D$ se réduire drastiquement. Par exemple, la part de la R\&D financée par le secteur privé en Pologne est passé de $52 \%$ en 1992 à $19 \%$ en 2007. A l'inverse des pays comme le Mexique, le Portugal et dans une moindre mesure le Danemark ont vu la contribution du secteur privé à l'effort de R\&D augmenter très significativement. Ainsi, la part de la $R \& D$ financée par le secteur privé au Mexique est passé de 14\% en 1993 à 45\% en 2007.

Par rapport aux cibles fixées par l'Union Européenne à l'horizon 2020 concernant l'investissement privé en $R \& D$, seuls trois pays de I'Union Européenne en 2007 étaient très proches ou au-dessus de ces objectifs, à savoir, l'Allemagne, la Suède et la Finlande. A noter que l'augmentation importante de la $R \& D$ financée par le secteur privé au Danemark rapproche également ce pays de ces objectifs.

\section{Dynamique du soutien financier à la R\&D privée au sein de l'OCDE}

Le tableau 3, qui représente l'évolution du montant du soutien financier direct en pourcentage du PIB, montre une tendance assez nette dans les pays industrialisés notamment. En effet, dans la majorité des pays développés ${ }^{19}$, les aides directes en pourcentage du PIB ont nettement reculé sur la période. D'ailleurs, si on représente le montant de ces aides directes en pourcentage de la R\&D financée par le secteur privé (tableau 4), cette tendance dans les pays industrialisés apparaît encore plus nettement. Concernant les autres pays de notre échantillon, la situation est assez hétérogène. Certains pays comme la Corée, la République tchèque ou l'Autriche ont assez nettement augmenté le budget des mesures de soutien direct sur la période alors que d'autres comme la Pologne l'ont fortement réduit(de $0.27 \%$ du PIB en 1992 à $0.02 \%$ en 2007). Notons que pour un certain nombre de pays, on voit une relative stabilité de la dotation des aides directes exprimée en pourcentage du PIB (Grèce, Hongrie, Irlande, Finlande, Belgique et Japon).

Concernant le soutien financier indirect, il émerge une tendance inverse à celle du soutien public direct mais qui s'étend au-delà des pays développés. Comme on peut le voir sur le graphique 5 qui représente la subvention fiscale moyenne par $\$$ investi en R\&D (obtenu en calculant 1-Bindex, voir annexe 2 pour des précisions), la majorité des pays de l'échantillon (16 exactement) ont significativement augmenté la générosité de leur système fiscal pour la R\&D. Seule l'Australie a réduit significativement la générosité de son système fiscal concernant la $R \& D$ sur la période. A l'heure actuelle, les pays les plus généreux en la matière sont le Portugal, l'Espagne, la France, la République Tchèque, la Norvège et dans une moindre mesure le Canada et la Hongrie. II est également intéressant de remarquer que

\footnotetext{
${ }^{19}$ Royaume-Uni, Suède, Norvège, Pays-Bas, France, Italie, Danemark, Etat-Unis, Allemagne et Canada
} 
les pays où l'intensité de la $R \& D$ privée est la plus forte ne sont pas les pays qui ont les systèmes fiscaux les plus avantageux ou sont même parfois des pays qui n'ont pas mis en place de mesures fiscales avantageuses (Suède, Finlande, Allemagne).

Si l'ensemble des pays de notre échantillon utilisent les instruments de soutien financier direct pour soutenir la R\&D privée, cela n'est pas le cas pour les instruments de soutien financier indirect. Cependant, comme le souligne Mohnen et Lokshin (2009), ces derniers sont tout de même de plus en plus utilisés au sein de l'OCDE puisque seulement 12 pays offraient des incitations fiscales à la R\&D en 1996 contre 21 en $2008^{20}$. Par ailleurs, parmi les pays utilisant un mix de mesures directes et indirectes, il semble émerger une tendance à la substitution des aides directes au profit des aides indirectes (surtout dans les pays développés).

\section{Dynamique de la R\&D exécutée par l'Etat au sein de l'OCDE}

Le tableau 6 qui représente les dépenses de R\&D exécutées par l'Etat (hors dépenses de $R \& D$ de l'enseignement supérieur) en pourcentage du PIB montre une tendance assez nette puisque sur les 25 pays de notre échantillon, 14 ont vu l'intensité de la R\&D exécutée par l'Etat diminuer significativement alors que pour 7 autres elle a stagné. Ainsi, la Suède, le Japon, l'Espagne et la Belgique, qui ont vu cette intensité augmenter sur la période, représentent des cas particuliers. Cette tendance pourrait traduire une volonté de substituer en partie la dotation des centres de recherche publics au profit de la dotation des mesures de soutien financier à la R\&D privée (et notamment les mesures fiscales).

\section{Dynamique du taux d'intérêt nominal à long terme au sein de l'OCDE}

Comme nous pouvons le voir sur le graphique 7 , le taux d'intérêt nominal à long terme a connu une baisse structurelle sur la période pour l'ensemble des pays de notre échantillon. Quatre pays ont connu des baisses drastiques, à savoir, le Mexique, la Grèce, la Pologne et la Hongrie. En effet, dans ces quatre pays, le taux d'intérêt nominal était supérieur ou égal à $30 \%$ au début des années 90 alors qu'à la fin des années 2000 il était proche de $10 \%$.

\section{Dynamique des crédits distribués au secteur privé au sein de l'OCDE}

Le graphique 8 qui représente l'évolution des crédits intérieurs accordés au secteur privé montre une forte progression de ces derniers pour l'ensemble des pays à l'exception du Japon, du Mexique, de la Finlande et de la République Tchèque pour lesquels les crédits accordés au secteur privé n'ont pas augmenté voir reflué.

\footnotetext{
${ }^{20}$ Voir Thomson (2009) pour une revue des mesures fiscales en place dans les pays de notre échantillon
} 


\section{Methodologie et résultats}

\subsection{Methodologie}

Nickell (1981) a montré que l'estimation d'un modèle dynamique sur données de panel par les estimateurs OLS (Ordinary Least Squares) et LSDV (Least Squares Dummy Variable) est biaisée lorsque $N \rightarrow \infty$ et $T$ fixe car la variable endogène décalée est corrélée avec le terme d'erreur. Comme le souligne Bond (2002), l'estimation du coefficient de la variable décalée $(\rho)$ est biaisée à la hausse pour l'estimateur OLS et à la baisse pour l'estimateur LSDV. Par conséquent, l'estimation préliminaire de ces modèles est importante car cela permet d'obtenir des bornes de valeurs pour $\rho$ et de discuter les résultats théoriques concernant la supériorité des estimateurs.

Depuis l'article de Nickell (1981), la littérature économétrique a développé de nombreux estimateurs consistants qui utilisent les méthodes des variables instrumentales (Anderson et Hsiao (1982)) et des moments généralisés (Arellano et Bond (1991), Arellano et Bover (1995), Blundell et Bond (1998)). L'estimateur à variables instrumentales proposé par Anderson et Hsiao (1982) consiste à estimer le modèle (5) en différence première en utilisant la variable expliquée retardée de deux périodes comme instrument. Cet estimateur est efficace lorsque $N \rightarrow \infty$ et $T$ fixe. Cependant comme le souligne Lai and al. (2008), les propriétés asymptotiques de cet estimateur ne sont plus valables pour de nombreuses configurations d'échantillon ce qui implique de fortes différences entre la performance asymptotique de l'estimateur et sa performance sur échantillon fini. Ceci est notamment le cas lorsque les instruments utilisés sont faibles ou en trop grand nombre. Un instrument est dit faible lorsque la contrainte sur les moments qu'il implique fournit peu d'information relativement à la taille de l'échantillon (Lai and al. (2008)). Ce problème d'instruments faibles est très important car comme le montre Bond et al. (1995), même avec un échantillon comportant un très grand nombre d'individus, l'estimateur de Anderson et Hsiao (1982) fournit des résultats très peu fiables lorsque les instruments sont faibles.

D'autres estimateurs sont basés sur la méthode des moments généralisés. De manière similaire à l'estimateur d'Anderson et Hsiao (1982), ces estimateurs ont de bonnes propriétés lorsque $N \rightarrow \infty$ et $T$ fixe et sous condition que les instruments utilisés ne soient pas faibles. On distingue deux types d'estimateurs GMM (Generalized Method of Moments) pour les modèles dynamiques sur données de panels, à savoir, l'estimateur GMM en différence et l'estimateur GMM en système. Le premier estime le modèle (5) en différence première en utilisant comme instruments les variables décalées en niveau alors que le second estime un système d'équations à la fois en différence première et en niveau en utilisant comme instruments dans les équations en niveau, les différences premières des variables décalées. Comme le montre Arellano et Bover (1995) et Blundell et Bond (1998), lorsque les données 
sont fortement persistantes et que le nombre de périodes est faible, l'estimateur GMM en différence fournit de mauvaises estimations car dans ces conditions, les variables décalées en niveau constituent de faibles instruments. Blundell et Bond (1998) montrent la supériorité de l'estimateur GMM en système dans ce cas. Ainsi, il apparaît plus pertinent d'estimer notre modèle avec un estimateur GMM en système qu'avec un estimateur GMM en différence du fait des spécificités de notre échantillon et de la forte persistence de l'intensité des dépenses privées de R\&D dans le temps.

Une autre voie pour fournir une estimation efficace des modèles dynamiques sur données de panel consiste à corriger le biais de l'estimateur LSDV (Nickell 1981, Kiviet 1995, Kiviet 1999, Bun and Kiviet 2003). L'avantage de cette méthode est double puisque d'une part, l'estimateur LSDV a souvent une variance plus faible que les autres estimateurs et d'autre part, une correction du biais de l'estimateur LSDV permet de fournir une estimation consistante pour l'ensemble des dimensions de panels. D'ailleurs, les simulations Monte-Carlo réalisées par Kiviet (1995), Kiviet (1999), Bun and Kiviet (2003) et Bruno (2005a) montrent la supériorité de l'estimateur LSDV corrigé (LSDVC) par rapport aux estimateurs IV et GMM que ce soit en termes de biais et de RMSE (Root Mean Squared Error). Bruno (2005b) a développé une extension de l'estimateur LSDVC de Bun and Kiviet (2003) aux données non cylindrées. Cependant, contrairement aux estimateurs précédents qui permettent une estimation efficace en présence de régresseurs endogènes, les estimateurs LSDVC suppose a minima une exogénéité faible. L'estimateur LSDVC proposé par Bruno (2005b) suppose une stricte exogénéité des régresseurs du modèle. Dans notre cas, nous pouvons soupçonner a minima que certaines variables peuvent être faiblement endogènes comme les mesures de soutien direct et indirect à la R\&D.

Par conséquent, les estimateurs potentiels ont tous des avantages et des inconvénients étant donné la dimension de notre panel et de notre objet d'étude. Afin d'éliminer les estimateurs non efficaces, nous avons réalisé des estimations du modèle (5) par les estimateurs OLS et LSDV afin de déterminer des bornes de $\rho$ puis estimer ce modèle à l'aide de l'estimateur proposé par Anderson et Hsiao (1982) en différence et en niveau (AH-D et AH-N), l'estimateur GMM d'Arellano-Bond (GMM-AB), l'estimateur GMM de Bundell et Bond (1998) ainsi que l'estimateur LSDVC de Bruno (2005a). Nous avons également réalisé des tests de sur-identification afin de mettre en avant la présence d'instruments faibles. Les résultats de ces estimations et les tests de sur-identification de Sargan et de Hansen sont présentés dans le tableau 4 de l'annexe 5.

Les estimations OLS et LSDV nous montrent que la valeur de $\rho$ doit être comprise entre 0,862 et 0,985 . L'estimateur AH-D fournit une valeur de $\rho$ inférieure à celle de l'estimateur LSDV si bien que nous excluons cet estimateur. L'estimateur $\mathrm{AH}-\mathrm{N}$ fournit quant à lui une valeur de $\rho$ très légèrement supérieure à celle fournit par l'estimateur LSDV. Les tests de Sargan effectués sur ces deux estimateurs ne nous permettent pas de conclure sur la 
validité des instruments utilisés pour deux raisons. En effet, ce test n'est pertinent que si les erreurs sont homoscédastiques et si le nombre d'instruments utilisés n'est pas trop élevé. Dans notre cas, l'hypothèse d'homoscédasticité des erreurs paraît forte et le nombre d'instruments utilisés par ces estimateurs est trop grand ce qui explique une $p$-value de 0 pour le test de Sargan. De ce fait, en se basant sur les valeurs de $\rho$ estimées par les estimateurs $\mathrm{AH}-\mathrm{D}$ et $\mathrm{AH}-\mathrm{N}$, nous excluons ces estimateurs comme candidats.

L'estimation du modèle (5) à l'aide de l'estimateur GMM-AB est fourni en colonne 5 du tableau 4. La valeur estimée de $\rho$ est inférieure à celle de l'estimateur LSDV. Par ailleurs, les valeurs prises par les tests de Sargan et Hansen montrent que ces derniers ne sont pas pertinents du fait de l'utilisation d'un nombre trop important d'instruments. Cependant, la réduction du nombre d'instruments ne permet pas d'améliorer la qualité de cet estimateur qui fournit alors des estimations peu précises et une valeur de $\rho$ encore plus faible. Par conséquent, nous éliminons l'estimateur d'Arellano-Bond comme candidat. Dans la colonne 6, l'estimateur GMM-BB estime la valeur $\rho$ à 0,886 qui est comprise entre sa valeur estimée par l'estimateur OLS et l'estimateur LSDV. Encore une fois, les tests de Hansen et de Sargan ne sont pas interprétables du fait d'un trop grand nombre d'instruments. La réduction du nombre d'instruments augmente très légèrement la valeur estimée de $\rho$ mais fournit des estimations moins précises (forte variance des paramètres estimés). Lorsque nous réduisons très fortement le nombre d'instruments jusqu'à un niveau inférieur au nombre de pays inclus dans nos données ${ }^{21}$, la valeur estimée de $\rho$ dépasse 1. Ainsi, même si les tests de sur-identification effectués ne nous permettent pas d'être certain de la validité des instruments utilisés, nous conservons l'estimateur GMM-BB qui est le seul à fournir une estimation valable de $\rho$ parmi l'ensemble des estimateurs vus jusqu'ici.

Finalement, l'estimation du modèle (5) à l'aide de l'estimateur LSDVC est fournie en colonne $7 \mathrm{du}$ tableau 4. La valeur de $\rho$ estimée est supérieure à celle de l'estimateur GMMBB et compris dans l'intervalle des possibles. Par conséquent, nous retenons cet estimateur et l'estimateur GMM-BB pour notre étude qui sont les deux seuls à fournir une estimation plausible de $\rho$.

\footnotetext{
${ }^{21}$ Selon Roodman (2006), la littérature fournit peu d'information pour connaître le nombre maximum d'instruments à utilisés. Une règle minimale est d'avoir un nombre d'instrument inférieur au nombre d'individus.
} 


\subsection{Résultats}

\subsubsection{Impact et complémentarité des politiques internes de soutien financier à la R\&D}

Les colonnes 1 et 2 du tableau 5 (annexe 5) présentent l'estimation du modèle (5). Comme la théorie le suggère, nos résultats font apparaître une forte persistance de l'intensité des dépenses privées de R\&D au cours du temps. Sa vitesse d'ajustement par rapport à son niveau cible est ainsi estimée entre 9 et $11 \%$ ce qui montre une réactivité potentielle des décisions d'investissements en $R \& D$ aux changements économiques très limitée à court terme. Ainsi, l'effet de court terme des variables exogènes sera beaucoup plus faible que leur effet à long terme.

Concernant les variables "financières", nos résultats font ressortir une forte influence du taux d'intérêt et dans une moindre mesure du volume de crédits accordés au secteur privé. Ainsi, l'élasticité au taux d'intérêt de court (long) terme est comprise entre $-0.068(-0.78)$ et $-0.075(-0.66)$. Si les crédits accordés au secteur privé influencent positivement l'intensité de la R\&D privée, cet impact n'apparaît statistiquement significatif qu'avec l'estimateur GMM-BB. D'ailleurs, si la sensibilité de l'intensité de la R\&D privée aux volumes de crédit est similaire à celle du taux d'intérêt selon l'estimateur GMM-BB, elle est beaucoup plus faible pour l'estimateur LSDVC. Ces résultats montrent néanmoins une influence significative des conditions de financement sur l'investissement en R\&D des firmes.

Nos résultats ne font pas apparaître d'influence significative de la $R \& D$ publique (hors universités) sur l'intensité des dépenses de $R \& D$ du secteur privé. Ce résultat est nettemment différent de ceux proposés par Guellec et al. (2003) ou Shin (2006) qui trouvaient un impact négatif et positif respectivement. Nous pensons que ces différences de résultats sont liées au fait que Guellec et al. (2003) analyse l'influence de la R\&D publique sur le taux de croissance des dépenses de R\&D privée alors que nous étudions son impact sur l'intensité des dépenses privées de R\&D. Concernant l'effet positif mis en avant par Shin (2006), il peut-être lié au fait que leur mesure de la $R \& D$ publique intègre les dépenses de $R \& D$ réalisées par l'enseignement supérieur qui ont un effet positif marqué chez Guellec et al. (2003).

Concernant l'influence des politiques de soutien financier à la $R \& D$ privée, nos résultats montrent un effet positif et significatif des mesures fiscales alors qu'aucun effet significatif des mesures de soutien direct apparaît. La sensibilité à court (long) terme de l'intensité de la $R \& D$ privée au B-index est comprise entre $-0.11(-1.31)$ et $-0.16(-1.37)$. Pour comparaison, Falk (2006) et Shin (2006) estiment une élasticité proche de -1 à long terme. Ainsi, notre résultat vient confirmer le résultat de Falk (2006) sur la capacité des mesures fiscales à augmenter significativement l'intensité des dépenses privées de R\&D. Cependant, 
le B-index étant une mesure globale de la générosité du système fiscal et notre étude étant menée au niveau macroéconomique, nous ne pouvons pas utiliser l'élasticité estimée pour mesurer la capacité de ces mesures à générer un effet de levier sur l'investissement privé en R\&D.

Nos résultats ne montrent pas d'influence significative des mesures de soutien direct sur l'intensité de la R\&D privée contrairement à ceux de Guellec et al. (2003) ou Shin (2006) qui soutiennent l'idée d'un effet significativement positif. Comme nous l'avons précédemment indiqué, l'utilisation d'une mesure relative des aides directes permet de mieux contrôler l'effet-prix sur les inputs de la R\&D qui biaise à la hausse l'estimation du coefficient du soutien public direct dans les articles précités. D'ailleurs notre résultat est identique à celui proposé par Falk (2006). Ce résultat qui rend compte d'une certaine neutralité des politiques de soutien financier direct sur l'intensité de la R\&D financée par le secteur privé ne traduit pas forcément un impact négatif de ces politiques. II signifie simplement que les dotations des politiques de soutien financier direct vont augmenter l'intensité de la R\&D d'un pays via l'augmentation de l'intensité de la $R \& D$ financée par le secteur public mais n'exerceront pas d'effet significatif sur l'intensité de la R\&D financée par le secteur privé.

Une question importante qui se posent pour les autorités publiques qui utilise un mix de mesures de soutien direct et indirect est celle de la complémentarité entre les différents instruments. Puisque les instruments de soutien direct et indirect reposent sur des mécanismes incitatifs différents, on peut imaginer qu'ils rentrent en conflit les uns avec les autres. Les résultats de Guellec et al. (2003) montrent d'ailleurs que mesures directes et indirectes ne sont pas complémentaires pour augmenter l'investissement privé en R\&D puisqu'il apparaît que l'augmentation des mesures de soutien direct (indirect) réduit l'effet positif des mesures de soutien indirect (mesures de soutien direct) sur l'investissement privé en R\&D.

Pour étudier l'intéraction entre instruments de soutien direct et indirect, nous estimons une extension du modèle (5) qui introduit une variable croisée des politiques de soutien financier à la R\&D nommée INTERACT ${ }^{22}$. Les résultats des estimations de cette extension du modèle (5) sont présentés dans les colonnes 3 et 4 du tableau 5 . Ils confirment le résultat proposé par Guellec et al. (2003) et montrent que les instruments de soutien direct et indirect sont des substituts pour dynamiser l'intensité de la R\&D privée. Cependant, contrairement à Guellec et al. (2003), dans notre cas le coefficient estimé de la variable SUBPIB est négatif (et devient significatif lorsqu'on introduit la variable INTERACT). Cela signifie donc qu'une augmentation des mesures fiscales à la $R \& D$ va augmenter l'effet négatif des subventions et qu'une augmentation des mesures de soutien direct va réduire l'effet positif des mesures fiscales. Ainsi, les instruments de soutien direct et indirect semblent rentrer en conflit pour augmenter l'intensité de la R\&D financée par le secteur privé. Notons cependant que cet

\footnotetext{
${ }^{22}$ La variable INTERACT représente donc le produit de la variable SUBPIB et de la variable BINDEX.
} 
effet de substitution entre instruments n'est statistiquement significatif qu'avec l'estimateur LSDVC.

A l'image des résultats proposés par Falk (2006), nos résultats confirment la capacité des instruments de soutien indirect à inciter les firmes à accroître leur investissement en R\&D alors que cela ne semble pas être le cas concernant les instruments de soutien direct (qui n'exercent pas pour autant un effet de substitution). Ces différents instruments semblent en revanche entrer en conflit pour augmenter l'intensité de la $R \& D$ privée ce qui met en avant la nécessité d'avoir une approche globale pour la définition d'un policy mix. Nos résultats font également ressortir l'influence significative des conditions de financement (volume de crédits et taux d'intérêt) ce qui laisse penser que des actions pour améliorer ces conditions aurait un impact significatif sur l'investissement privé en R\&D. Finalement, l'absence d'influence de la R\&D publique (hors enseignement supérieur) nous laisse penser que cette dernière n'entraîne pas d'effets externes très significatifs sur les entreprises.

\subsubsection{Importance des effets externes entre pays de l'OCDE}

Afin d'analyser les intéractions entre politiques nationales de soutien à la $R \& D$ et plus généralement l'influence des conditions extérieures sur les décisions d'investissements en $R \& D$ des firmes dans un pays, nous réalisons des estimations du modèle (7). Comme nous l'avons précisé précedemment, nous utilisons différentes matrices de pondération spatiale afin de tester différentes formes de proximité. Le tableau 6 présente les estimations réalisées sur le modèle (7). Les acronymes WCOM, WDIST et WBREV renvoient aux matrices de pondération spatiale basées sur le commerce bilatéral, sur la distance géographique et sur les collaborations technologiques respectivement.

A partir des résultats présentés dans le tableau 6 de l'annexe 6 , nous pouvons remarquer que l'extension du modèle (5) au modèle (7) ne modifie pas les résultats précédemment obtenus. En effet, les coefficients des variables exogènes (non décallées spatialement) sont de même signe et de même ampleur que ceux présentés dans le tableau 5.

On remarque également que les estimations utilisant la matrice de pondération basée sur les collaborations dans les demandes de brevets PCT (colonne 5 et 6 du tableau 6 ) ne font pas ressortir d'influence des variables exogènes décalées spatialement sur l'intensité de la R\&D privée. Ainsi, cette matrice de proximité ne fait pas apparaître d'impact des conditions extérieures sur les décisions d'investissement en R\&D d'un pays. Ce résultat assez surprenant $a$, selon nous, différentes explications. La première est que la proximité technologique influence l'intensité des dépenses privées de R\&D par le biais d'autres variables que

celle que nous avons retenus. La deuxième est que les collaborations dans les demandes de 
brevets $\mathrm{PCT}$ (qui ne constituent qu'une petite partie des demandes de brevets) ne reflètent pas correctement les interactions technologiques entre pays. La dernière est que la proximité technologique n'est pas une bonne mesure pour capter l'influence des conditions extérieures sur l'intensité des dépenses privées de R\&D d'un pays.

Les estimations du modèle (7) utilisant la matrice spatiale basée sur le commerce bilatéral sont présentées dans les colonnes 1 et 2 du tableau 6. Les estimations réalisées à partir des estimateurs GMM-BB et LSDVC montrent que la R\&D publique menée par les partenaires commerciaux d'un pays influence très positivement l'intensité des dépenses privées de $R \& D$. Cet effet positif est important puisque l'élasticité de court (long) terme est comprise entre $0.472(4.9)$ et $0.524(6)$. Ce résultat est d'autant plus intéressant que parallèlement nos résultats ne montrent pas d'influence significative de la $R \& D$ publique nationale. Cela suggère que c'est davantage la quantité globale de connaissances qui produit des effets d'externalités significatifs sur la $R \& D$ privée que la production de connaissances d'un pays en particulier.

L'estimateur LSDVC montre également une influence négative du volume de crédits accordés au secteur privé des partenaires commerciaux. Cela signifie que plus les crédits accordés au secteur privé chez les partenaires commerciaux du pays $i$ sont importants plus l'intensité de la R\&D privée dans ce pays sera faible. Cette relation montre l'importance de l'attractivité exercée par les facilités d'accès au financement.

Les estimations réalisées en utilisant la matrice spatiale basée sur la distance géographique sont présentées dans les colonnes 3 et 4 du tableau 6 . On peut remarquer qu'à l'image des estimations précédentes, la R\&D publique menée par les pays voisins semble exercer un effet positif sur l'intensité des dépenses privées de R\&D. Nos résultats montrent également une influence du taux d'intérêt et du niveau d'aides publiques directes des pays voisins. L'estimateur GMM-BB rend compte d'un impact positif du taux d'intérêt moyen pondéré des pays voisins alors que l'estimateur LSDVC montre un impact positif du niveau des aides publiques directes à la $R \& D$ des pays voisins. Le résultat concernant le taux d'intérêt va dans le sens de notre remarque concernant l'impact des crédits accordés au secteur privé chez les principaux partenaires commerciaux d'un pays, à savoir, une influence significative des conditions de financements extérieurs au pays.

Les estimations réalisées sur le modèle (7) fournissent plusieurs résultats intéressants concernant l'influence des conditions et politiques de soutien financier extérieures sur l'investissement privé en $R \& D$ d'un pays. Le premier est que les mesures de soutien direct et indirect extérieures à un pays n'influencent pas significativement l'intensité de la R\&D privée dans ce pays. Cela tend à confirmer les résultats de la littérature sur la localisation de la R\&D qui montrent une importance très secondaire des politiques de soutien financier dans le choix de la localisation de la R\&D (Hall, 2011). D'ailleurs, le fait que le B-index soit significatif mais pas son décallage spatial (quelle que soit la matrice de proximité utilisée) 
nous laisse penser à une efficacité beaucoup plus forte de ces mesures sur les PME que sur les grandes entreprises. En effet, ce sont prioritairement le grandes firmes qui peuvent modifier la localisation de leur activités de $R \& D$ et donc jouer sur les écarts de fiscalité. Selon nous, ce résultat renforce ceux de la littérature montrant une efficacité plus forte des mesures fiscales sur les PME que sur les grandes entreprises (voir Mohnen et Loshkin, 2009). Ces éléments soulignent également la faiblesse de l'argument selon lequel ces outils (et notamment les mesures fiscales) doivent être utilisés pour attirer la R\&D. D'autant plus que le signe du coefficient estimé du décallage spatial du B-index est négatif ce qui traduirait (s'il était significatif) une complémentarité entre politiques fiscales à la R\&D.

Nos résultats font également ressortir une influence forte du niveau de $R \& D$ publique menée par les partenaires commerciaux et les pays voisins ce qui suggère que les effets d'externalités de la $R \& D$ publique sur la $R \& D$ privée se produisent davantage via le stock global de connaissances produites que par le stock national de connaissances produites. Nos estimations font également ressortir l'influence des conditions de financements extérieures sur l'intensité de la R\&D privée (bien que ces effets soient moins nets). Ainsi, un pays ayant des conditions de financements plus difficiles que ces voisins et partenaires commerciaux aura un handicap supplémentaire pour doper les investissements privés en R\&D.

\section{Conclusion}

Les pays de l'OCDE dépensent un montant considérable de fonds publics pour soutenir financièrement les activités privée de R\&D. Un des objectifs de ces politiques est d'inciter les entreprises à augmenter leur investissement en R\&D. Dans cet article, nous avons essayé de comprendre l'impact macroéconomique des politiques de soutien financier direct et indirect sur l'intensité de la R\&D financée par le secteur privé. Pour analyser leur effets internes et externes, nous avons estimés différents modèles empiriques d'investissement privé en $R \& D$ sur des données de panel couvrant 25 pays de l'OCDE sur la période 1990-2007.

Nos résultats font apparaître une différence nette entre l'impact des mesures de soutien direct et celui des mesures de soutien indirect. Les premières ne semblent pas avoir d'impact significatif alors que les secondes ont un effet positif important. Cette différence d'impact prend encore plus de sens lorsque l'on s'intéresse à la question d'un effet complémentaire de ces différents types d'instrument. En effet, comme chez Guellec et al. (2003), nos résultats montrent que les mesures directes sont des substituts aux mesures indirectes (et vice versa) pour augmenter l'intensité de la R\&D privée. Cela signifie que la mise en place d'une mesure de soutien direct (indirect) supplémentaire va augmenter les effets d'aubaines des 
mesures indirectes (directes) en place. Ce dernier résultat met en avant la nécessité pour les pouvoirs publics d'avoir une approche globale dans la définition d'un mix de mesures directes et indirectes.

Nous avons également cherché à comprendre dans quelle mesure les conditions économiques extérieures et plus spécifiquement les politiques extérieures de soutien financier influencent l'intensité de la R\&D privée d'un pays. Nos résultats ne montrent pas d'influence significative des politiques extérieures de soutien à la $R \& D$ mais font ressortir un rôle fortement positif du niveau de R\&D publique menées par les partenaires commerciaux et les pays voisins. Ces résultats soutiennent donc l'idée que le niveau de soutien public à la $R \& D$ privée n'influence pas significativement la localisation des activités de R\&D mais influence la dynamique interne de la $R \& D$ privée. Par ailleurs, l'influence positive de la $R \& D$ publique extérieure alors que la $R \& D$ publique interne n'a pas d'influence significative sur l'intensité de la R\&D privée suggère que ce sont davantage les connaissances produites par la $R \& D$ publique au niveau mondial qu'au niveau national qui génèrent des effets externes positifs sur la R\&D privée.

Ainsi, dans un strict objectif d'augmentation de l'intensité de la R\&D financée par le secteur privé, nos résultats et ceux de la littérature penchent davantage pour des mesures de soutien indirect. Cependant, comme le montre le survey de Mohnen et Lokshin (2009), si les mesures de crédit d'impôt incrémental exercent un effet de levier sur l'investissement privé en $R \& D$, cela ne semble pas être le cas des mesures de crédit d'impôt en volume. De la même façon, l'efficacité des mesures fiscales apparaît beaucoup plus important sur les PME que sur les grandes entreprises. Ces éléments soulignent donc l'importance du design des mesures fiscales à la $R \& D$ (et plus généralement des instruments financiers de soutien à la R\&D) et mettent en avant les mesures fiscales ciblées qui réduisent les effets d'aubaines en ciblant davantage les bénéficiaires tout en conservant leur capacité à générer un effet de levier sur l'investissement privé en R\&D. 


\section{Bibliographie}

Anderson T. \& Hsiao C. (1982), "Formulation and estimation of dynamic models using panel data", Journal of Econometrics, vol.18, p.570-606

Arellano M. \& Bond S. (1991), "Some tests of specification for panel data : Monte Carlo evidence and an application to employment equations", Review of Economic Studies, vol.58, p.277-297

Bérubé C., Mohnen P., Canada B., Chabot R. \& Thornton G. (2007), "Are Firms That Received R\&D Subsidies More Innovative?", Industrial Research, vol. 42, Issue 1, p. 206-225

Bond S., Hoeffler A. \& Temple J. (2001), "GMM estimation of Empirical Growth Models", Economics Papers N²001-W21, University of Oxford

Bond S. (2002), "Dynamic Panel Data Models : a guide to micro data methods and practice", Portuguese Economic Journal, vol.1, p. 141-162

Bloom N., Griffith R. \& Van Reenen J. (2002), "Do R\&D tax credit works? Evidence from a panel of countries 1979-1997", Journal of Public Economics, vol.85, p.1-31

Blundell R. \& Bond S. (1998), "Initial conditions and moment restrictions in dynamic panel data models", Journal of Econometrics, vol.97, p.115-143

Bruno G. (2005a), "Estimation and inference in dynamic unbalanced panel-data models with a small number of individuals", The Stata Journal, vol.5, n4, p.473-500

Bruno G. (2005b), "Approximating the bias of the LSDV estimator for dynamic unbalanced panel data models", Economics Letters, vol.87, p.361-366

Bun M. \& Carre M. (2005), "Bias-corrected estimation in dynamic panel data models", Journal of Business and Economic Statistics, vol.23, n², p.200-210

Capron H. \& Van Pottelsberghe de la Potterie B. (1997), "Public Support to Business R\&D : A survey and some new quantitative evidence", in Policy Evaluation in Innovation and Technology, OECD

Carvalho A. (2011), "Why are tax incentives increasingly used to promote private R\&D ?", CEFAGE-UE Working Paper, Universidade de Evora

Corchuelo B. \& Martinez-Ros E. (2009), "The Effects of Fiscal Incentives for R\&D in Spain", Working Paper 09-23, Business Economic Series 02, Université de Madrid 
Czarnitski D., Hanel P. \& Rosa J. (2011), "Evaluating the impact of R\&D tax credits on innovation: A microeconometric study on Canadian Firms", Research Policy, vol.40, p. $217-229$

David P., Hall B. \& Toole A. (2000), "Is public R\&D a complement or substitute for private R\&D? A review of the econometric evidence", Research Policy, n²9, p.497-529

David P. \& Hall B. (2000), "Heart of darkness : modeling public-private funding interactions inside the R\&D black box", Research Policy, n²9, p.1165-1183

Falk M. (2006), "What drives business Research and Development (R\&D) intensity across Organisation for Economic Co-operation and Development (OECD) countries?", Applied Economics, vol.38, p.533-547

Garcia-Quevedo J. (2004), "Do Public Subsidies Complement Business R\&D? A MetaAnalysis of the Econometric Evidence", KYKLOS, vol.57, p.87-102

Görg H. \& Strobl E. (2007), "The Effect of R\&D Subsidies on private R\&D", Economica, vol.74, p.215-234

Guellec D. \& Van Pottelsberghe de la Potterie B. (2003), "The Impact of Public R\&D Expenditure on Business R\&D", Economics of Innovation and New Technology, vol.12, p. $225-243$

Hall B. \& Van Reenen J. (2000), "How effective are fiscal incentives for R\&D? A review of the evidence", Research Policy, n²9, p.449-469

Hall B. (2011), "The internationalization of R\&D", working paper $N^{\circ} 49$, UNU-MERIT, United Nations University

Judson R. \& Owen A. (1999), "Estimating dynamic panel data models : a guide for macroeconomists", Economics Letters, vol.65, p.9-15

Kiviet J. (1995), "On bias, inconsistency, and efficiency of various estimators in dynamic panel data model", Journal of Econometrics, vol.68, p.53-78

Lai T., Small D. \& Liu J. (2008), "Statistical Inference in dynamic panel data models", Journal of statistical planning and inference, vol. $138, n^{\circ} 9$, p.2763-2776

Lentile D. \& Mairesse J. (2009), "A policy to boost R\&D : Does the R\&D tax credit work?", EIB PAPERS, vol.14, p.143-169

Lichtenberg F.R (1987), "The Effect of Government Funding on Private Industrial Reserach and Development : A Re-assessment", Journal of Industrial Economics, vol.36, p.97104 
McFetridge, D.G \& Warda J. (1983), "Canadian Tax incentive: Their Adequacy and Impact", Canadian Tax Paper n70, Canadian Tax Foundation

Mohnen P. \& Lokshin B. (2009), "What does it take for an R\&D tax incentive policy to be effective?", working paper for the CEPR project "SCience, Innovation, FIrms and markets in a GLObalized World (SCl-FI GLOW)"

OECD (2006), "Science, Technology and Industry Outlook 2006", p.242

Roodman D. (2009), "How to do xtabond2: An introduction to difference and system GMM in Stata", The Stata Journal, vol.9, N¹, p. 86-136

Shin T. (2006), "Behavioural Additionality of Public R\&D Funding in Korea", in OECD, Government R\&D Funding and Company Behaviour: Measuring Behavioural Additionality, chap. 9, p.167-180

Thomson R.(2009), "Tax Policy and the Globalisation of R\&D", Australian National University, Working Paper $\mathrm{n}^{\circ} 3$

Van Pottelsberghe de la Potterie B., Nysten S. \& Megally E. (2003), "Evaluation of current fiscal incentives for business R\&D in Belgium", Université Libre de Bruxelles, Working paper.

Warda J. (2005), "Measuring the Value of R\&D Tax Provisions: A Primer on the B-index for Analysis and Comparisons", Paper prepared for The OMC Working Group on "Design and evaluation of fiscal measures to promote business research, development and innovation"

Warda J. (2006), "Tax treatment of business investments in intellectual assets: an international comparison", STI Working Paper n4, DSTI OECD

Wolff G. \& Reinthaler V. (2008), "The effectiveness of subsidies revisited : Accounting for wage an employment effects in business R\&D", Research Policy, vol.37, p.1403-1412 


\section{Annexes}

Annexe 1 : Cible d'investissement en R\&D dans les pays de l'OCDE

\begin{tabular}{|c|c|c|c|}
\hline Table C.1 & \multicolumn{3}{|c|}{ R\&D targets and expenditures, selected OECD countries ${ }^{a}$} \\
\hline Country/region & $\begin{array}{r}R \& D \text { intensity } \\
\text { in } 2005\end{array}$ & Target & Target date and characteristics \\
\hline Austria & 2.36 & 3.00 & 2010 \\
\hline Belgium & 1.82 & 3.00 & 2010 \\
\hline Canada & 1.98 & Top 5 OECD & 2010 \\
\hline Cyprus & 0.40 & 1.00 & 2010 \\
\hline Czech Republic & 1.42 & 2.06 & $\begin{array}{l}\text { Target of } 1 \% \text { public R\&D with estimated } \\
1.06 \% \text { of private expenditure in } 2010\end{array}$ \\
\hline Denmark & 2.44 & 3.00 & Target of $1 \%$ public R\&D in 2010 \\
\hline Estonia & 0.94 & 1.90 & 2010 \\
\hline Finland & 3.48 & 4.00 & 2010 \\
\hline France & 2.13 & 3.00 & 2010 \\
\hline Germany & 2.51 & 3.00 & 2010 \\
\hline Greece & 0.61 & 1.50 & 2010 \\
\hline Hungary & 0.94 & $1.80 \mathrm{Inc}$ & ncreased participation of private sector by 2010 \\
\hline Ireland & 1.25 & 2.50 & 2013 \\
\hline Italy & 1.10 & 2.50 & 2010 \\
\hline Korea & 2.99 & Double spending & 2007 \\
\hline Latvia & 0.57 & 1.50 & 2010 \\
\hline Lithuania & 0.76 & 2.00 & 2010 \\
\hline Luxembourg & 1.56 & 3.00 & 2010 \\
\hline Malta & 0.61 & 0.75 & 2010 \\
\hline Netherlands & 1.78 & 3.00 & 2010 \\
\hline New Zealand & 1.14 & & $\begin{array}{c}\text { Target of OECD average }(0.68 \%) \text { for public } \\
\text { R\&D - no year specified }\end{array}$ \\
\hline Norway & 1.51 & 3.00 & $\begin{array}{l}\text { Target of } 1 \% \text { public and } 2 \% \text { private R\&D by } \\
2010\end{array}$ \\
\hline Poland & 0.57 & 1.65 & 2008 \\
\hline Portugal & 0.81 & 1.80 & $\begin{array}{c}\text { Target of } 1 \% \text { public R\&D and tripling of } \\
\text { private R\&D by } 2010\end{array}$ \\
\hline Slovenia & 1.22 & 3.00 & 2010 \\
\hline Slovakia & 0.51 & 1.80 & 2010 \\
\hline Spain & 1.12 & 2.00 & 2010 \\
\hline Sweden & 3.86 & 4.00 & $\begin{array}{c}\text { Target of } 1 \% \text { public R\&D and unchanged } \\
\text { private R\&D by } 2010\end{array}$ \\
\hline Turkey & 0.66 & 2.00 & $\begin{array}{l}\text { Target of } 1 \% \text { public R\&D and } 1 \% \text { private R\&D } \\
\text { by } 2010\end{array}$ \\
\hline United Kingdom & 1.79 & 2.50 & 2014 \\
\hline
\end{tabular}

a Values for Italy, United Kingdom and the Netherlands are for 2004, New Zealand are for 2003, and Turkey are for 2002.

Data sources: Council of the European Union 2006, Eurostat Science and Technology Database 2007, http://epp.eurostat.ec.europa.eu, (accessed February 2007)), OECD 2004c, 2006c New Zealand Ministry of Research, Science \& Technology Budget Speech (May 2006). 


\section{Annexe 2 : Présentation du B-index}

Le B-index est une mesure synthétique de la générosité du système fiscal national de la R\&D développée par McFetridge et Warda (1983). Cet indice renvoie au rendement minimum avant impôt nécessaire d'un investissement marginal en R\&D pour qu'il devienne rentable de réaliser cet investissement. Mathématiquement, le B-index est égal au coût après incitations fiscales d'un investissement d'un dollar en R\&D divisé par un moins le taux d'imposition sur les bénéfices :

$$
\text { B-index }=\frac{1-A}{1-\tau}
$$

où $\tau$ est le taux d'imposition sur les bénéfices et $A$ représente la valeur nette actualisée des déductions fiscales, crédits d'impôts et mesures d'amortissements spécifiques sur les actifs de la R\&D. La valeur de $A$ est composée de trois éléments principaux : la valeur nette actualisée des déductions fiscales $A_{d}$, la valeur nette actualisée des mesures d'amortissements $A_{s}$ et la valeur nette actualisée des mesures de crédits d'impôts $A_{c}$. Ainsi, en tenant compte de la proportion des coûts de la $R \& D$ éligibles à ces mesures notée respectivement $\left(p_{d}, p_{s}, p_{c}\right)$, on détermine la valeur nette actualisée de l'ensemble des mesures fiscales à la R\&D notée $A$.

En termes d'interprétation, plus le traitement fiscal de la R\&D d'un pays est favorable plus le $\mathrm{B}$-index est faible. Ainsi, lorsque $B<1$ cela indique que les pouvoirs publics subventionnent fiscalement les dépenses de R\&D des firmes. A l'inverse si $B>1$ cela signifie que le revenu avant taxes lié à un investissement marginal en $R \& D$ ne permet pas de supporter l'ensemble des coûts et taxes induit par cet investissement. Ce dernier cas de figure apparaît lorsque les firmes doivent payer des impôts sur les revenus générés par l'investissement en R\&D (Warda, 2006). Pour résumer, le modèle du B-index considère que le système fiscal à la $R \& D$ d'un pays offre une subvention fiscale positive lorsque plus de $100 \%$ des dépenses de $R \& D$ peuvent être déduites, une subvention nulle lorsque $100 \%$ des dépenses sont déductibles et une subvention négative si les dépenses de R\&D ne peuvent pas être déduite à $100 \%$ dans l'exercice fiscal en cours. Notons qu'en calculant la valeur $1-B$-index, on obtient une mesure de la subvention fiscale reçue par dollar investi en $R \& D$.

La construction du B-index est basée sur une série d'hypothèses explicitées par McFetridge et Warda (1983) qui permettent d'utiliser cette mesure pour réaliser des comparaisons internationales sur la générosité du système fiscal à la $R \& D$. Nous évoquons ici les plus importantes d'entre elles et nous renvoyons le lecteur vers Warda (2005) et Thomson (2009) pour en avoir une description complète et exhaustive. Le B-index est construit sur la base d'une firme représentative qui est supposée avoir un ratio d'investissement en R\&D constant dans le temps et supposée réaliser des profits suffisant pour bénéficier au maximum des dispositions fiscales en vigueur. Ainsi, le B-index ne donne qu'une appréciation globale de la fiscalité sur la R\&D. 
Annexe 3 : Statistiques descriptives et corrélations

\begin{tabular}{c|ccc} 
Date & Freq. & Percent & Cum. \\
& & & \\
\hline 1990 & 15 & 3.51 & 3.51 \\
1991 & 20 & 4.68 & 8.20 \\
1992 & 21 & 4.92 & 13.11 \\
1993 & 23 & 5.39 & 18.50 \\
1994 & 23 & 5.39 & 23.89 \\
1995 & 25 & 5.85 & 29.74 \\
1996 & 25 & 5.85 & 35.60 \\
1997 & 25 & 5.85 & 41.45 \\
1998 & 25 & 5.85 & 47.31 \\
1999 & 25 & 5.85 & 53.16 \\
2000 & 25 & 5.85 & 59.02 \\
2001 & 25 & 5.85 & 64.87 \\
2002 & 25 & 5.85 & 70.73 \\
2003 & 25 & 5.85 & 76.58 \\
2004 & 25 & 5.85 & 82.44 \\
2005 & 25 & 5.85 & 88.29 \\
2006 & 25 & 5.85 & 94.15 \\
2007 & 25 & 5.85 & 100.00 \\
& & & \\
\hline Total & 427 & 100.00 &
\end{tabular}

Tableau 1 : Nombre de pays inclus par année (distribution du non cylindrage)

\begin{tabular}{l|ccccc}
\multicolumn{1}{c|}{ Variable } & Obs & Mean & Std. Dev. & Min & Max \\
\hline & & & & & \\
dirdefipib & 427 & $0,947 \%$ & $0,649 \%$ & $0,029 \%$ & $2,963 \%$ \\
dirdpubpib & 427 & $0,241 \%$ & $0,101 \%$ & $0,071 \%$ & $0,561 \%$ \\
subpib & 427 & $0,078 \%$ & $0,067 \%$ & $0,000 \%$ & $0,448 \%$ \\
credit & 427 & $91,893 \%$ & $47,144 \%$ & $15,214 \%$ & $231,082 \%$ \\
interetlt & 427 & $7,738 \%$ & $5,609 \%$ & $1,003 \%$ & $45,746 \%$ \\
bindex & 402 & 0,951 & 0,107 & 0,57 & 1,08
\end{tabular}

Tableau 2 : Statistiques descriptives

\begin{tabular}{l|cccccc} 
& dirdefipib & subpib & dirdpubpib & credit & interetlt & bindex \\
\hline dirdefipib & 1 & & & & & \\
subpib & 0.5000 & 1 & & & & \\
dirdpubpib & 0.2802 & 0.3400 & 1 & & & \\
credit & 0.4809 & 0.1622 & 0.0663 & 1 & & \\
interetlt & -0.4093 & -0.1096 & -0.0945 & -0.5421 & 1 & \\
bindex & 0.1937 & 0.1733 & 0.0760 & -0.1165 & 0.1938 & 1
\end{tabular}

Tableau 3 : Corrélations entre les variables du modèle 


\section{Annexe 4 : Graphiques des données}

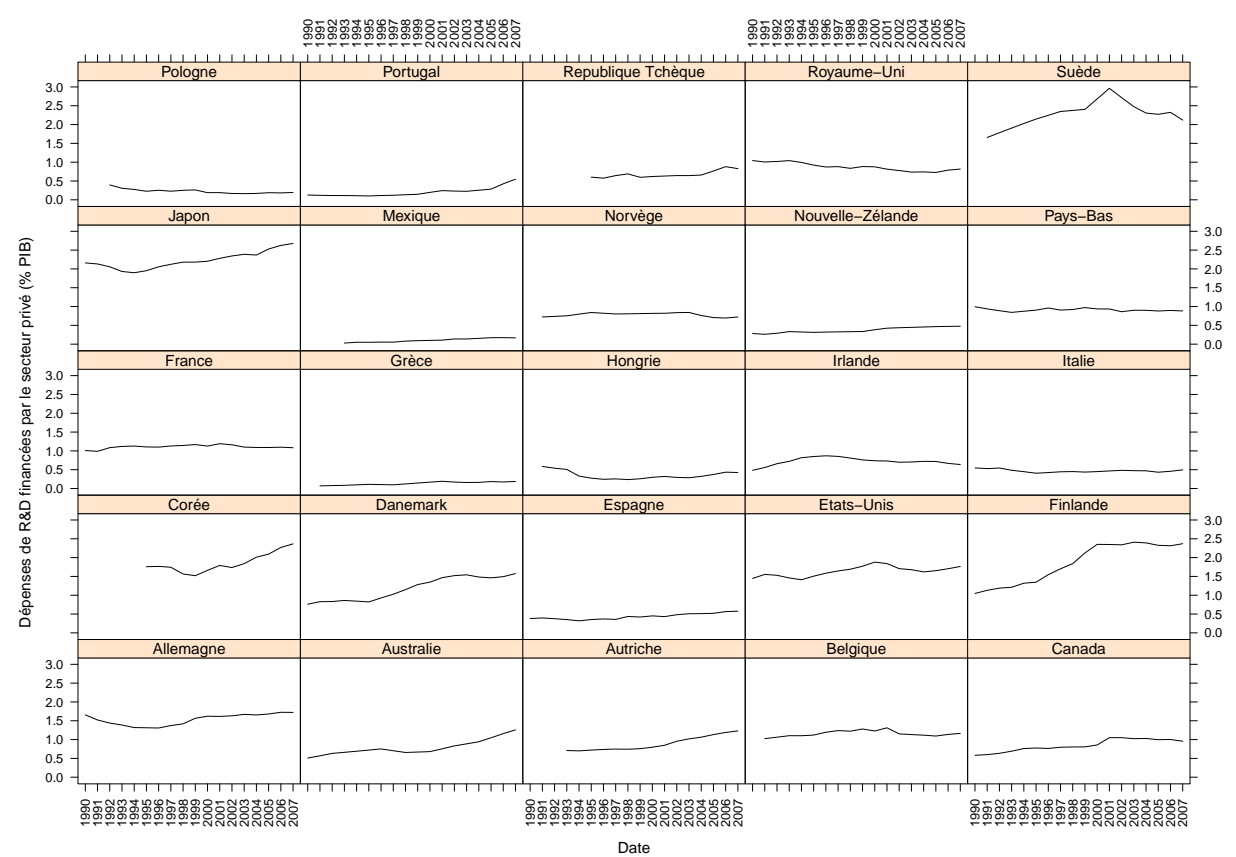

graphique 1 : Dépenses de R\&D financées par le secteur privé (\% du PIB)

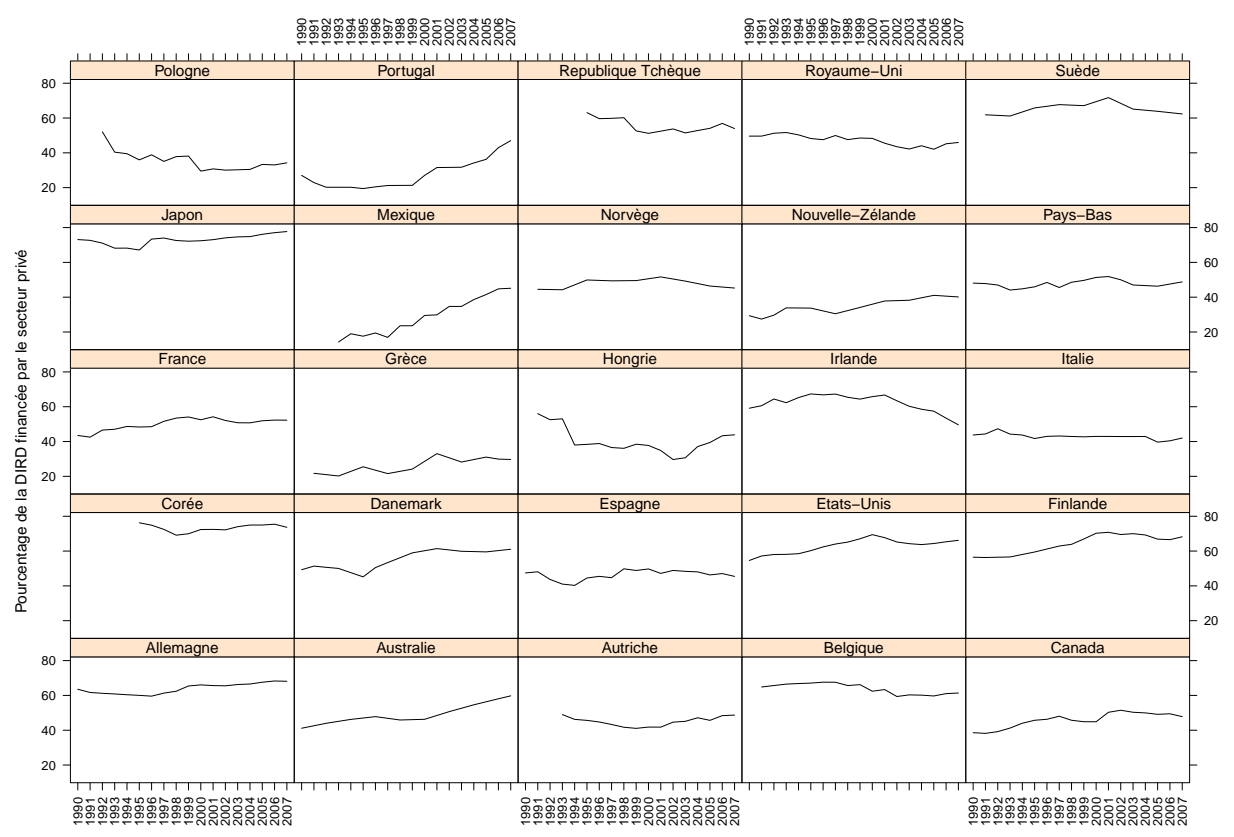

graphique 2 : Dépenses de R\&D financées par le secteur privé ( $\%$ de la DIRD) 


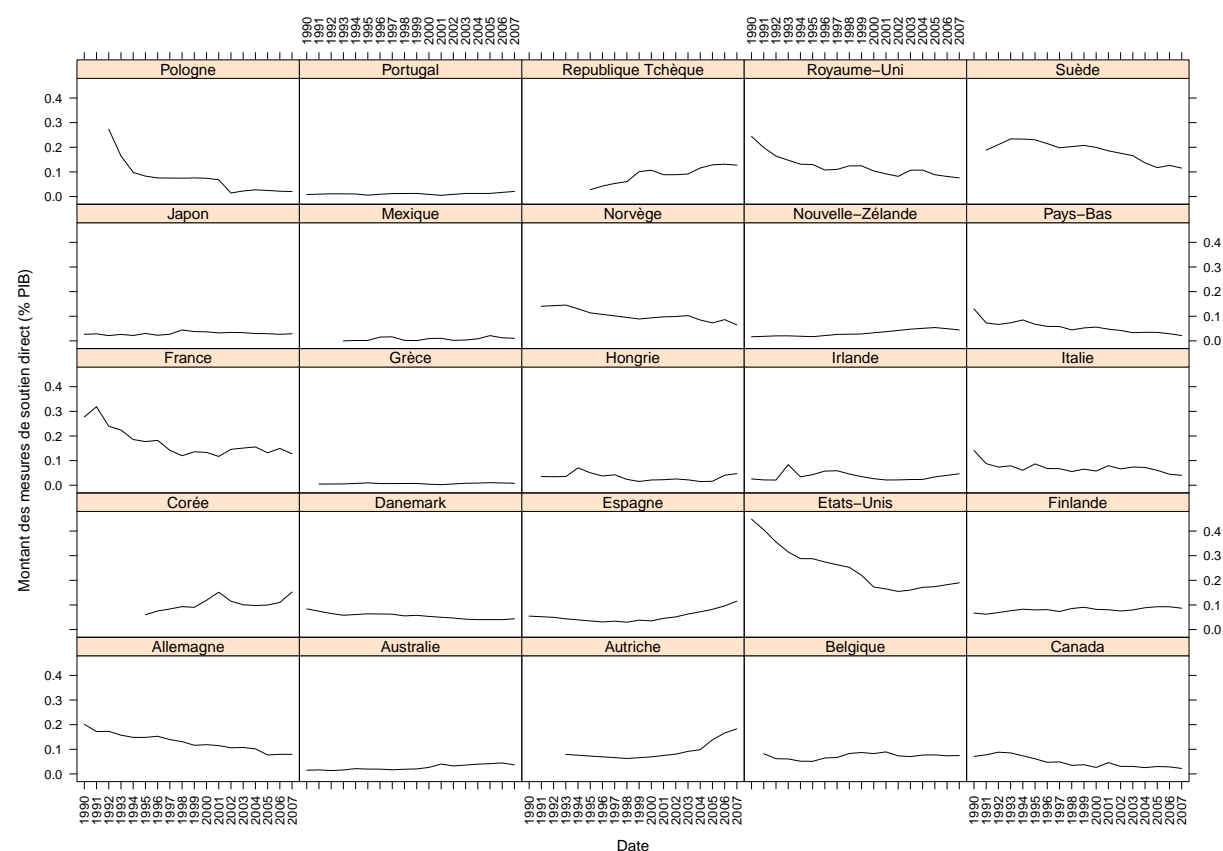

graphique 3 : Montant des mesures de soutien direct (\% du PIB)

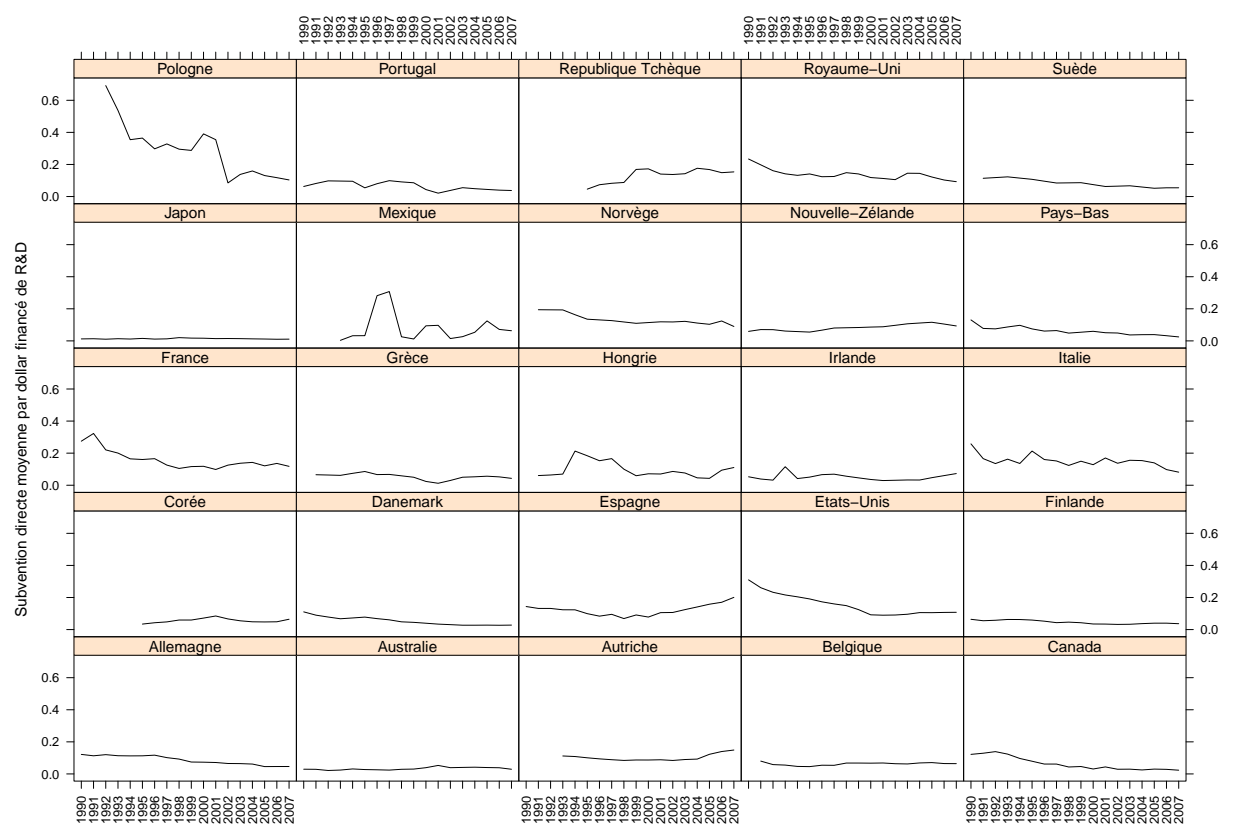

graphique 4 : Montant des mesures de soutien direct (\% de la R\&D financée par le secteur privé) 


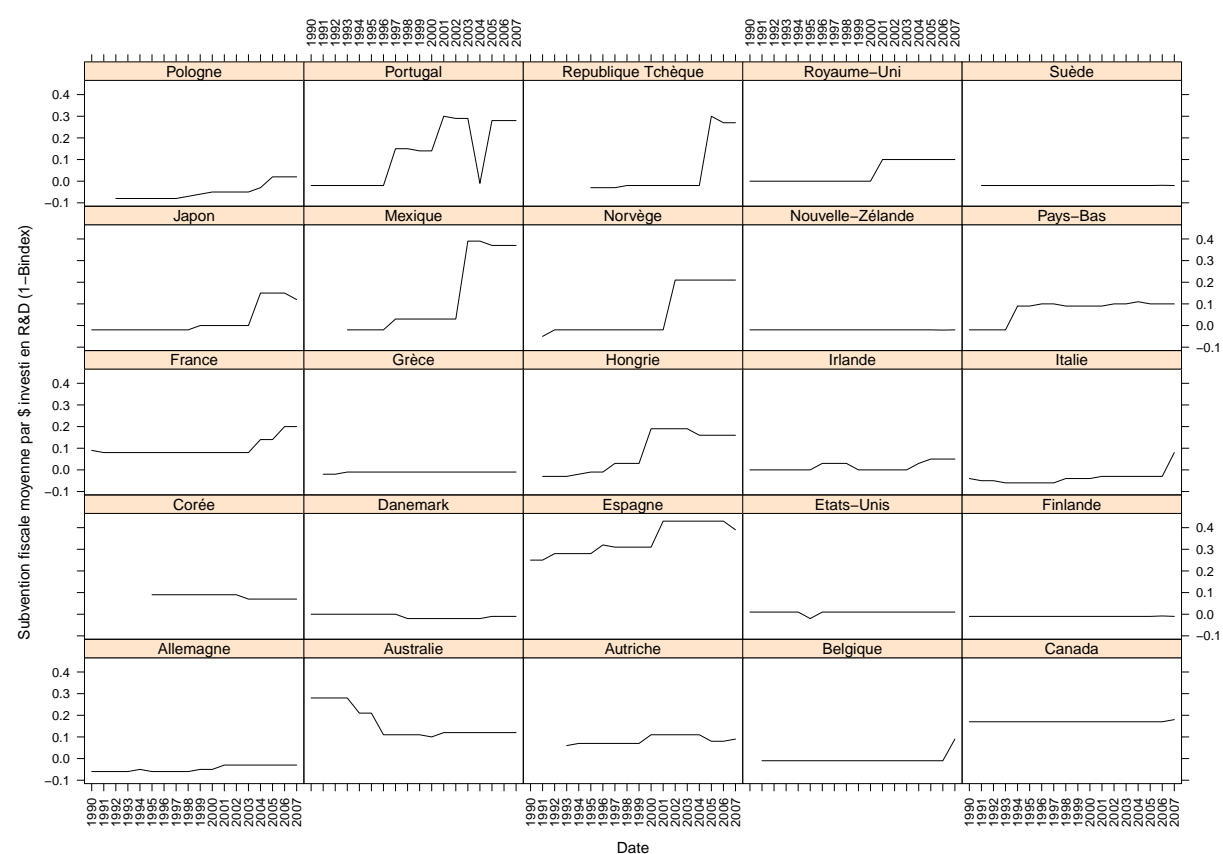

graphique 5 : Subvention fiscale par $\$$ investi en R\&D (1-Bindex)

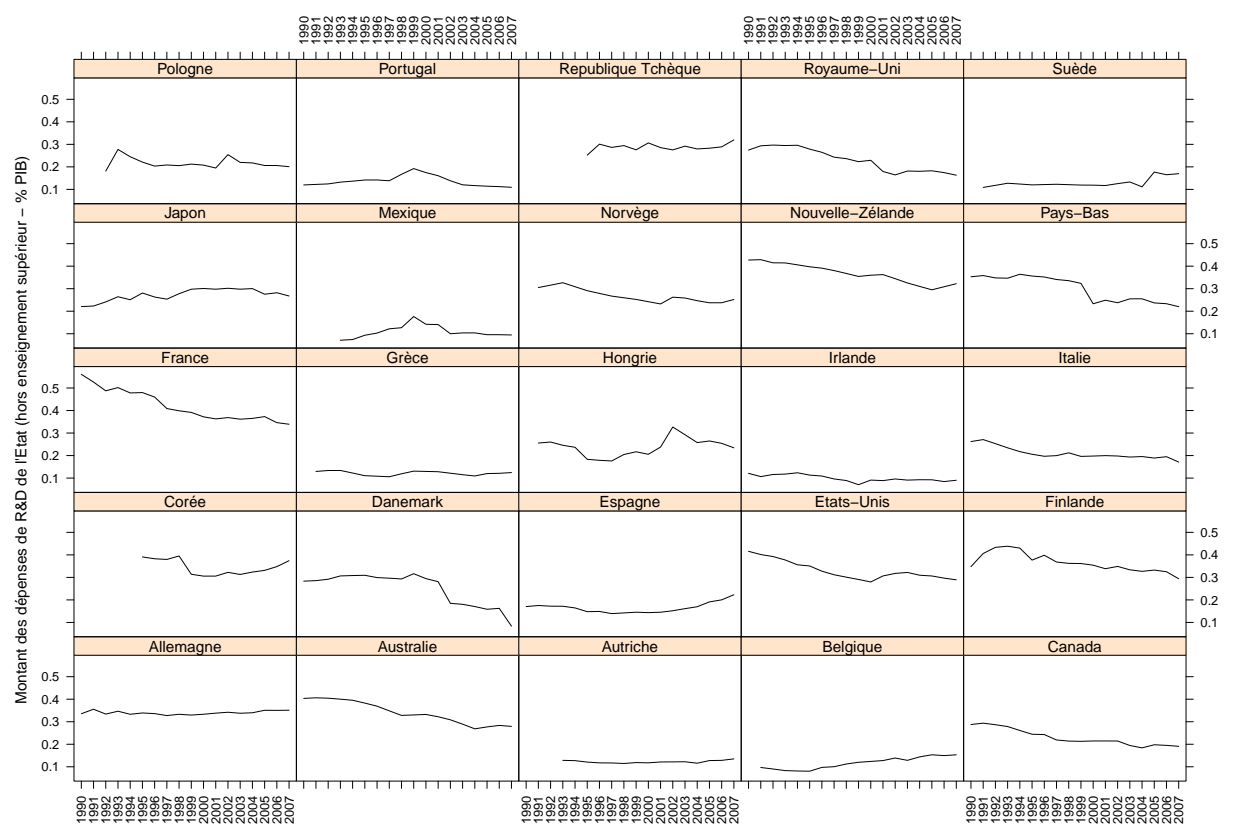

Date

graphique 6 : Dépenses de R\&D exécutées par l'Etat (hors enseignement supérieur - en \% du PIB) 


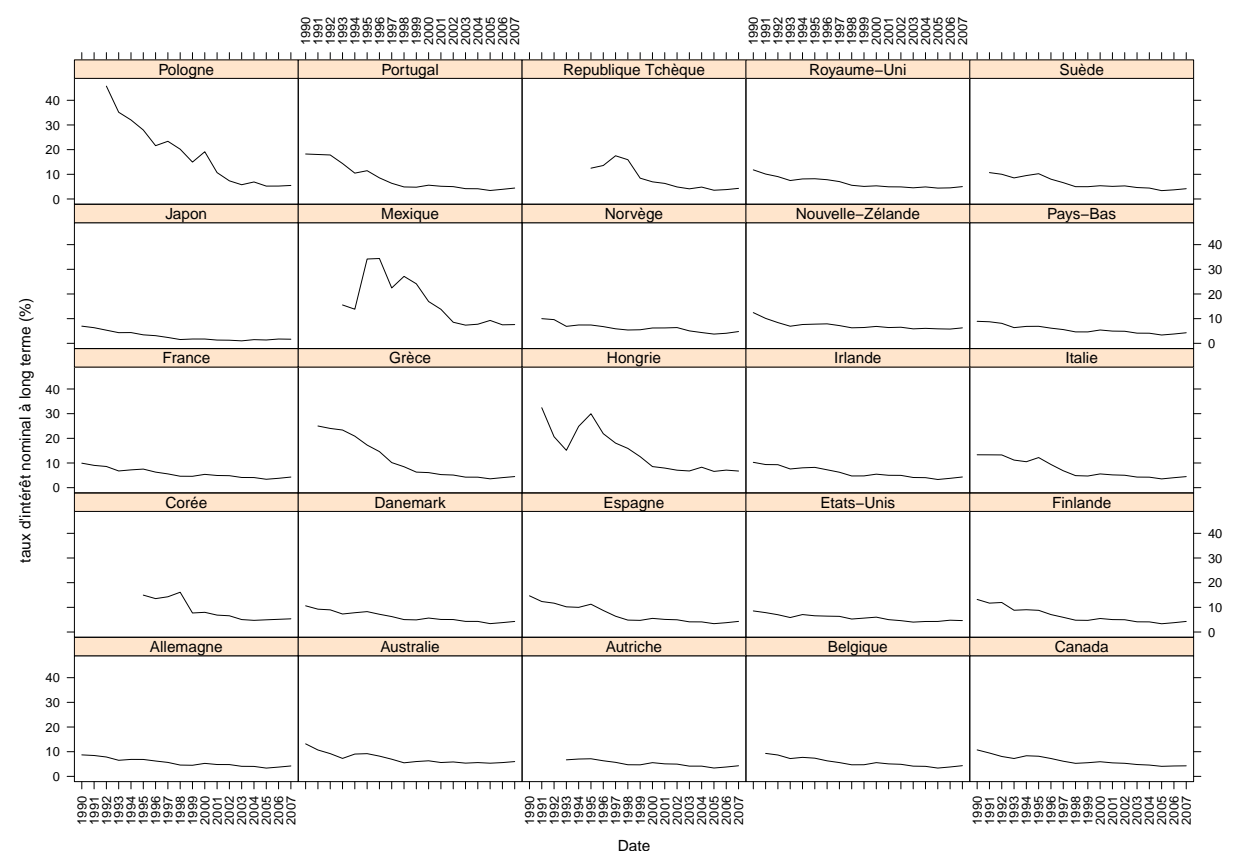

graphique 7 : Taux d'intérêt nominal à long terme (en \%)

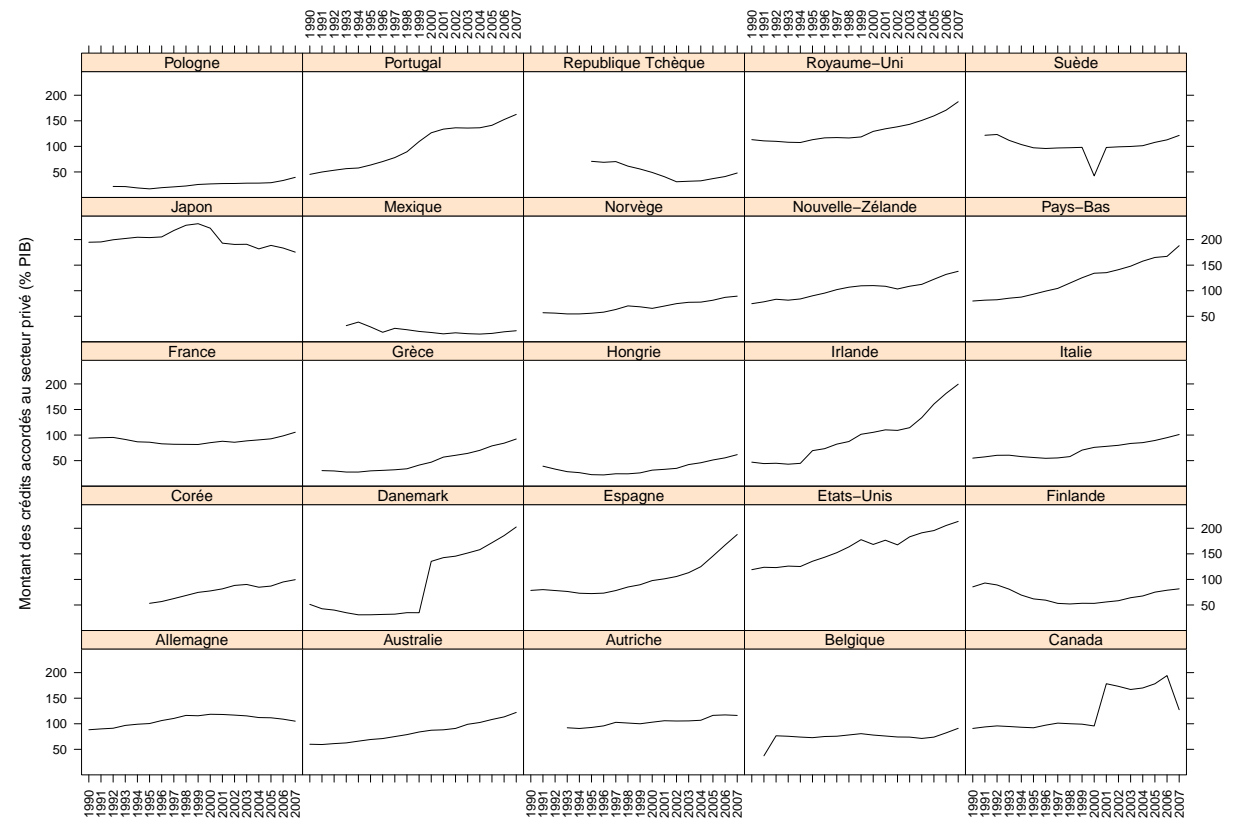

graphique 8 : Montant des crédits accordés au secteur privé (en \% du PIB) 


\subsection{Annexe 5: Tableaux de résultat}

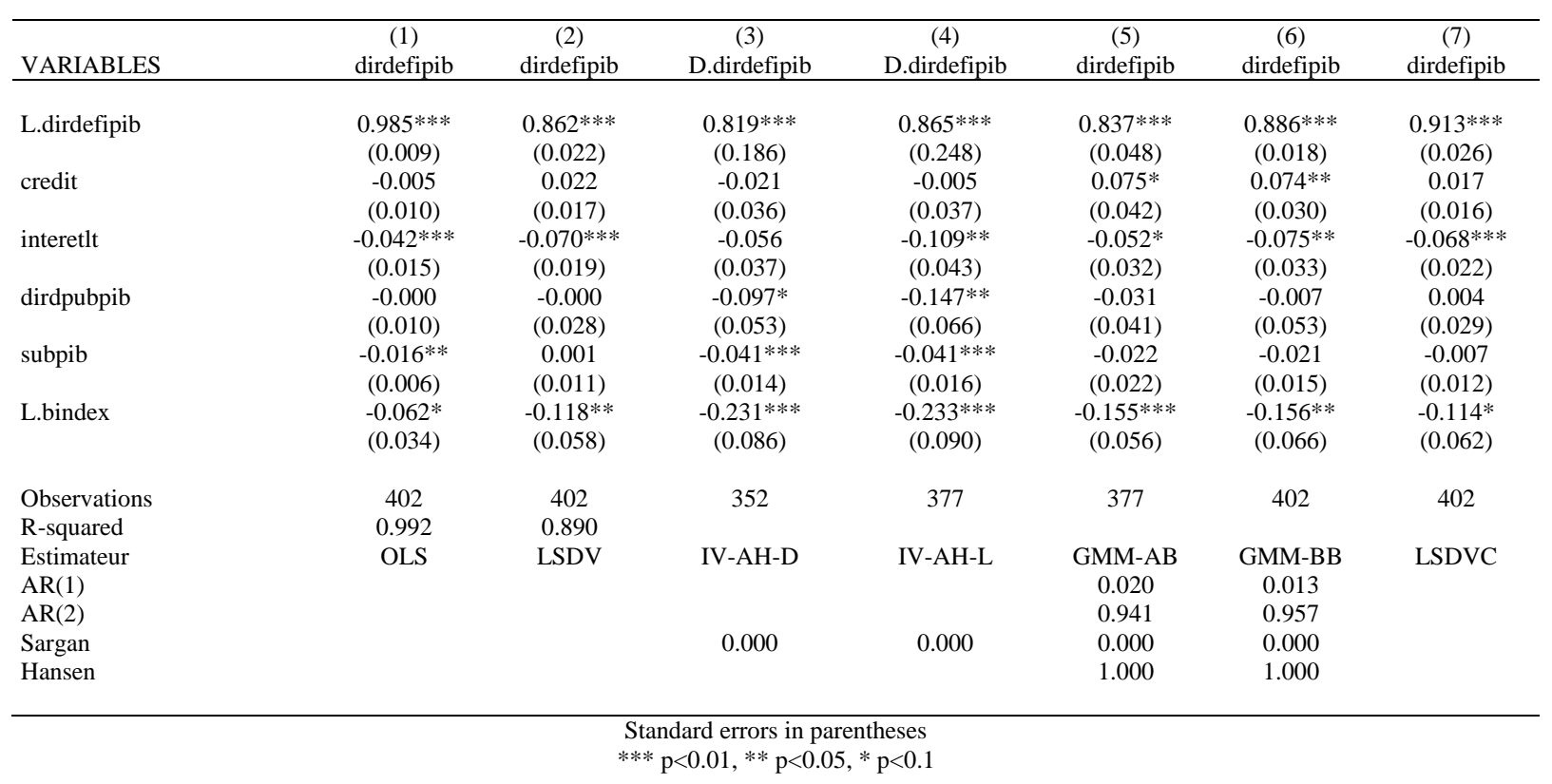

Tableau 4: Estimation du modèle (5)

Comparaison des estimateurs 


\begin{tabular}{|c|c|c|c|c|}
\hline VARIABLES & $\begin{array}{c}(1) \\
\text { dirdefipib } \\
\end{array}$ & $\begin{array}{c}(2) \\
\text { dirdefipib } \\
\end{array}$ & $\begin{array}{c}\text { (3) } \\
\text { dirdefipib }\end{array}$ & $\begin{array}{c}4) \\
\text { dirdefipib } \\
\end{array}$ \\
\hline L.dirdefipib & $\begin{array}{c}0.886^{* * * *} \\
(0.018)\end{array}$ & $\begin{array}{c}0.913 * * * \\
(0.026)\end{array}$ & $\begin{array}{c}0.885^{* * *} * \\
(0.018)\end{array}$ & $\begin{array}{c}0.904 * * * \\
(0.025)\end{array}$ \\
\hline credit & $\begin{array}{c}0.074 * * \\
(0.030)\end{array}$ & $\begin{array}{c}0.017 \\
(0.016)\end{array}$ & $\begin{array}{c}0.072 * * * \\
(0.027)\end{array}$ & $\begin{array}{c}0.010 \\
(0.016)\end{array}$ \\
\hline interetlt & $\begin{array}{c}-0.075^{* *} \\
(0.033)\end{array}$ & $\begin{array}{c}-0.068^{* * * *} \\
(0.022)\end{array}$ & $\begin{array}{c}-0.075^{* *} \\
(0.033)\end{array}$ & $\begin{array}{c}-0.056^{* *} \\
(0.023)\end{array}$ \\
\hline dirdpubpib & $\begin{array}{l}-0.007 \\
(0.053)\end{array}$ & $\begin{array}{c}0.004 \\
(0.029)\end{array}$ & $\begin{array}{l}-0.006 \\
(0.052)\end{array}$ & $\begin{array}{c}-0.005 \\
(0.028)\end{array}$ \\
\hline subpib & $\begin{array}{l}-0.021 \\
(0.015)\end{array}$ & $\begin{array}{l}-0.007 \\
(0.012)\end{array}$ & $\begin{array}{l}-0.026 \\
(0.018)\end{array}$ & $\begin{array}{c}-0.034 * * \\
(0.015)\end{array}$ \\
\hline L.bindex & $\begin{array}{c}-0.156^{* *} \\
(0.066)\end{array}$ & $\begin{array}{c}-0.114^{*} \\
(0.062)\end{array}$ & $\begin{array}{c}-0.162 * * \\
(0.064)\end{array}$ & $\begin{array}{c}-0.145^{* *} \\
(0.063)\end{array}$ \\
\hline interact & & & $\begin{array}{c}0.177 \\
(0.441)\end{array}$ & $\begin{array}{c}0.585^{* * * *} \\
(0.206)\end{array}$ \\
\hline Observations & 402 & 402 & 402 & 402 \\
\hline Estimateur & GMM-BB & LSDVC & GMM-BB & LSDVC \\
\hline $\mathrm{AR}(1)$ & 0.013 & & 0.013 & \\
\hline $\mathrm{AR}(2)$ & 0.957 & & 0.935 & \\
\hline Sargan & 0.000 & & 0.000 & \\
\hline Hansen & 1.000 & & 1.000 & \\
\hline
\end{tabular}

Table 5: Estimation modèle (5) Impact et complémentarité des politiques de soutien à la $R \& D$ privée 


\begin{tabular}{|c|c|c|c|c|c|c|}
\hline VARIABLES & $\begin{array}{c}(1) \\
\text { WCOM }\end{array}$ & $\begin{array}{c}(2) \\
\text { WCOM }\end{array}$ & $\begin{array}{c}\text { (3) } \\
\text { WDIST }\end{array}$ & $\begin{array}{c}\text { (4) } \\
\text { WDIST }\end{array}$ & $\begin{array}{c}\text { (5) } \\
\text { WBREV }\end{array}$ & $\begin{array}{c}(6) \\
\text { WBREV }\end{array}$ \\
\hline L.dirdefipib & $\begin{array}{c}0.903 * * * \\
(0.028)\end{array}$ & $\begin{array}{c}0.914 * * * \\
(0.026)\end{array}$ & $\begin{array}{c}0.885 * * * \\
(0.020)\end{array}$ & $\begin{array}{c}0.917 * * * \\
(0.026)\end{array}$ & $\begin{array}{c}0.877 * * * \\
(0.027)\end{array}$ & $\begin{array}{c}0.915 * * * \\
(0.028)\end{array}$ \\
\hline credit & $\begin{array}{c}0.069 * * \\
(0.034)\end{array}$ & $\begin{array}{c}0.008 \\
(0.016)\end{array}$ & $\begin{array}{c}0.076 * * \\
(0.032)\end{array}$ & $\begin{array}{c}0.014 \\
(0.017)\end{array}$ & $\begin{array}{c}0.092 * * \\
(0.038)\end{array}$ & $\begin{array}{c}0.009 \\
(0.019)\end{array}$ \\
\hline interetlt & $\begin{array}{c}-0.057^{*} \\
(0.033)\end{array}$ & $\begin{array}{c}-0.041^{*} \\
(0.022)\end{array}$ & $\begin{array}{c}-0.059^{*} \\
(0.031)\end{array}$ & $\begin{array}{c}-0.047 * * \\
(0.023)\end{array}$ & $\begin{array}{c}-0.079 * * * \\
(0.029)\end{array}$ & $\begin{array}{c}-0.062 * * * \\
(0.023)\end{array}$ \\
\hline dirdpubpib & $\begin{array}{c}0.006 \\
(0.042)\end{array}$ & $\begin{array}{c}0.049 \\
(0.033)\end{array}$ & $\begin{array}{c}0.045 \\
(0.039)\end{array}$ & $\begin{array}{c}0.031 \\
(0.035)\end{array}$ & $\begin{array}{l}-0.001 \\
(0.048)\end{array}$ & $\begin{array}{c}0.007 \\
(0.034)\end{array}$ \\
\hline subpib & $\begin{array}{l}-0.025 \\
(0.018)\end{array}$ & $\begin{array}{l}-0.018 \\
(0.012)\end{array}$ & $\begin{array}{l}-0.014 \\
(0.016)\end{array}$ & $\begin{array}{l}-0.011 \\
(0.012)\end{array}$ & $\begin{array}{l}-0.020 \\
(0.019)\end{array}$ & $\begin{array}{l}-0.007 \\
(0.013)\end{array}$ \\
\hline L.bindex & $\begin{array}{c}-0.139 * * * \\
(0.046)\end{array}$ & $\begin{array}{c}-0.166^{* * * *} \\
(0.062)\end{array}$ & $\begin{array}{c}-0.132 * * * \\
(0.046)\end{array}$ & $\begin{array}{c}-0.144^{* *} \\
(0.065)\end{array}$ & $\begin{array}{c}-0.110^{*} \\
(0.068)\end{array}$ & $\begin{array}{l}-0.103^{*} \\
(0.065)\end{array}$ \\
\hline wcredit & $\begin{array}{l}-0.077 \\
(0.139)\end{array}$ & $\begin{array}{c}-0.305^{* * *} \\
(0.114)\end{array}$ & $\begin{array}{c}0.126 \\
(0.238)\end{array}$ & $\begin{array}{l}-0.147 \\
(0.115)\end{array}$ & $\begin{array}{c}0.144 \\
(0.256)\end{array}$ & $\begin{array}{l}-0.305 \\
(0.195)\end{array}$ \\
\hline winteretlt & $\begin{array}{l}-0.016 \\
(0.072)\end{array}$ & $\begin{array}{c}0.018 \\
(0.063)\end{array}$ & $\begin{array}{l}0.256^{*} \\
(0.136)\end{array}$ & $\begin{array}{l}-0.004 \\
(0.092)\end{array}$ & $\begin{array}{c}0.062 \\
(0.274)\end{array}$ & $\begin{array}{l}-0.118 \\
(0.151)\end{array}$ \\
\hline wdirpubpib & $\begin{array}{c}0.472 * * \\
(0.206)\end{array}$ & $\begin{array}{c}0.524 * * * \\
(0.189)\end{array}$ & $\begin{array}{l}0.492^{*} \\
(0.257)\end{array}$ & $\begin{array}{l}0.375^{*} \\
(0.221)\end{array}$ & $\begin{array}{c}0.411 \\
(0.300)\end{array}$ & $\begin{array}{c}0.105 \\
(0.273)\end{array}$ \\
\hline wsubpib & $\begin{array}{l}-0.034 \\
(0.152)\end{array}$ & $\begin{array}{c}0.119 \\
(0.078)\end{array}$ & $\begin{array}{c}0.148 \\
(0.209)\end{array}$ & $\begin{array}{c}0.289 * * * \\
(0.082)\end{array}$ & $\begin{array}{l}-0.143 \\
(0.177)\end{array}$ & $\begin{array}{l}-0.019 \\
(0.115)\end{array}$ \\
\hline L.wbindex & $\begin{array}{l}-0.465 \\
(0.526)\end{array}$ & $\begin{array}{l}-0.305 \\
(0.307)\end{array}$ & $\begin{array}{l}-0.303 \\
(0.468)\end{array}$ & $\begin{array}{l}-0.172 \\
(0.434)\end{array}$ & $\begin{array}{c}1.192 \\
(1.428)\end{array}$ & $\begin{array}{c}0.101 \\
(0.684)\end{array}$ \\
\hline Observations & 402 & 402 & 402 & 402 & 402 & 402 \\
\hline Estimateur & GMM-BB & LSDVC & GMM-BB & LSDVC & GMM-BB & LSDVC \\
\hline $\mathrm{AR}(1)$ & 0.011 & & 0.013 & & 0.013 & \\
\hline $\operatorname{AR}(2)$ & 0.810 & & 0.866 & & 0.900 & \\
\hline Sargan & 0.000 & & 0.000 & & 0.000 & \\
\hline Hansen & 1.000 & & 1.000 & & 1.000 & \\
\hline
\end{tabular}

Tableau 6: Estimations du modèle (7) 